\title{
Uniformly distributed orbits of certain flows on homogeneous spaces
}

\author{
Nimish A. Shah \\ School of Mathematics, Tata Institute of Fundamental Research, Homi Bhabha Road, \\ Bombay 400005 , India
}

Received July 23, 1990

\section{Introduction}

Let $G$ be a connected Lie group, $\Gamma$ be a lattice in $G$ and $U=\left\{u_{t}\right\}_{t \in R}$ be a unipotent one-parameter subgroup of $G$, viz. Ad $u$ is a unipotent linear transformation for all $u \in U$. Consider the flow induced by the action of $U$ (on the left) on $G / \Gamma$. Such a flow is referred as a unipotent flow on the homogeneous space $G / \Gamma$. The study of orbits of unipotent flows has been the subject of several papers. For a nilpotent group $G$, a result of Green [13] implies that if $U$ has one dense orbit in $G / \Gamma$ then every orbit of $U$ is uniformly distributed with respect to a $G$-invariant measure on $G / \Gamma$. In the case when $G=S L(2, \mathbb{R})$, it was proved by Hedlund that every orbit of the unipotent (horocycle) flow is either dense or periodic; periodic orbits exist only when $G / \Gamma$ is non-compact. For a co-compact lattice, this result was strengthened by Fürstenberg [11] proving that every orbit is uniformly distributed with respect to a $G$-invariant measure. For non-uniform lattices in $S L(2, \mathbb{R})$, using a classification of invariant measures obtained by Dani in [2], Dani and Smillie [3] proved that every non-periodic orbit is uniformly distributed. There are also various results obtained on orbit closures and invariant measures etc. of larger subgroups consisting of unipotent elements, especially the horospherical subgroups. Recently, there was a spurt in the area initiated by Margulis' proof (cf. [15], see also [7]) of Oppenheim conjecture on values of quadratic forms at integral points using the study of unipotent flows. The reader is referred to the survey articles by Dani [4] and Margulis [14] for various related developments.

We now note some conjectures expected to hold for orbits of a unipotent flow, namely the $U$-action on $G / \Gamma$ as above (though we restrict to $U$ being a oneparameter subgroup, the first two conjectures are expected to hold for any subgroup generated by unipotent elements contained in it). A conjecture due to Raghunathan on orbit closures states the following:

Conjecture 1. For every $x \in G / \Gamma$, there exists a closed subgroup $F$ such that $\overline{U x}=F x$.

For $G=S L(2, \mathbb{R})$ the conjecture follows from the result of Hedlund mentioned above. It was recently verified for "generic" unipotent flows in the case of 
$G=S L(3, \mathbb{R})[8]$. Upto certain easy modifications, these are the only cases of semisimple groups where the conjecture is known to hold.

The following measure theoretic analogue of the above conjecture was recently proved by Ratner [19].

Conjecture 2. For any finite ergodic $U$-invariant measure $\sigma$ on $G / \Gamma$, there exists a closed subgroup $F$ containing $U$ and $x \in G / \Gamma$ such that $F x$ is closed and $\sigma$ is a $F$-invariant measure supported on $F x$.

In this paper we shall be concerned with the following conjecture which strengthens Conjectures 1 and 2.

Conjecture 3. For every $x \in G / \Gamma$, there exists a closed subgroup $F$ such that $F x$ is closed, $F x$ admits a $F$-invariant probability measure $\sigma$ and the $U$-orbit through $x$ is uniformly distributed with respect to $\sigma$; that is, for all bounded continuous functions $f$ on $G / \Gamma$,

$$
\lim _{T \rightarrow \infty} \frac{1}{T} \int_{0}^{T} f\left(u_{t} x\right) d t=\int_{G / \Gamma} f d \sigma .
$$

It may be observed that if the $U$-orbit of $x \in G / \Gamma$ is uniformly distributed with respect to the $F$-invariant probability measure on $F x$ as above then $\overline{U x}=F x$. Thus Conjecture 3 includes Conjecture 1 . Also since for any ergodic invariant measure there are "generic points" [10], Conjecture 3 is stronger than Conjecture 2.

Our object is to prove the following result on the asymptotic behaviour of the orbits of certain unipotent flows. Specifically, we choose $G$ to be a reductive Lie group and let $U$ be a regular unipotent one-parameter subgroup of $G$, in the sense that $U$ is contained in a unique maximal unipotent subgroup of $G$ (see Theorem 4.3 for other equivalent conditions) and prove the following.

(1.1) Main Theorem. Let $G$ be a connected reductive Lie group, $\Gamma$ be a lattice in $G$ and $U=\left\{u_{t}\right\}_{t \in \mathbb{R}}$ be a regular unipotent one-parameter subgroup of $G$. Let $X=G / \Gamma$ and let $Y$ be the subset of $X$ consisting of all points $y$ such that $F y$ is closed for some proper closed subgroup $F$ of $G$, containing $U$. Let $x \in X \backslash Y$. Then, given $\varepsilon>0$, there exists a compact subset $K \subset X \backslash Y$ such that for all $T>0$,

$$
\frac{1}{T} \ell\left\{t \in[0, T] \mid u_{t} x \in K\right\}>1-\varepsilon,
$$

where $\ell$ is the Lebesgue measure on $\mathbb{R}$.

Together with Ratner's classification of finite ergodic $U$-invariant measures, the theorem enables us to describe geometrically the set of points whose $U$-orbits are uniformly distributed with respect to a $G$-invariant measure; in particular, we are able to conclude the validity of Conjecture 3 for regular unipotent oneparameter subgroups when either $G / \Gamma$ is compact or the $\mathbb{R}$-rank of $[G, G]$ is 1 . The results may be stated as follows:

(1.2) Corollary. Let $G, \Gamma$, and $U$ be as in the Main theorem. Then for every $x \in X \backslash Y$, the $U$-orbit of $x$ is uniformly distributed with respect to the G-invariant probability measure on $G / \Gamma$. In particular, all these orbits are dense in $G / \Gamma$.

(1.3) Corollary. Let $G, \Gamma$, and $U$ be as in the Main theorem. Suppose further that $G / \Gamma$ is compact. Then Conjecture 3 holds (for all $x \in X$ ). 
(1.4) Corollary. Let $G$ be a connected reductive Lie group such that the $\mathbb{R}$-rank of $[G, G]$ is 1 . Let $\Gamma$ be a lattice in $G$ and $U$ be any unipotent one-parameter subgroup of $G$. Then Conjecture 3 holds (for all $x \in X$ ).

Like the earlier results on unipotent flows [14], the above results on uniformly distributed orbits raise certain interesting possibilities of application to Diophantine approximation. In particular, given a nondegenerate indefinite quadratic form $Q$ on $\mathbb{R}^{3}$ which is not a multiple of a rational quadratic form and $\varepsilon>0$ one can get lower estimates, for all larger $r \in \mathbb{R}$, for the number of solutions $x \in \mathbb{Z}^{3}$, $\|x\|<r$ for the inequality $|Q(x)|<\varepsilon$. On account of the somewhat incomplete nature of the results currently obtained and some new ideas involved in the proof, we shall deal with the applications elsewhere.

The method of proof of the Main theorem is an adaptation of the ideas developed in [3] and the Appendix of [8]. In this method one relates thin neighbourhoods of subsets of $Y$ as above to certain subsets of linear $G$-spaces and uses polynomial behaviour of orbits of one-parameter groups of unipotent linear transformations in vector spaces, to study properties of $U$-orbits on $G / \Gamma$.

The paper is organized as follows. In Sect. 2 we prove that if $x \in G / \Gamma$ and $F$ is the smallest closed subgroup of $G$ containing $U$ such that $F x$ is closed then $F x$ admits a finite $F$-invariant measure and the $U$-action on $F x$ is ergodic. This result, which would also be of general interest, is used in Sect. 3 to show that any $F$ as above comes from a special class of subgroups. The conclusion is used in Sect. 5 to give a geometric description of the set $Y$ defined as in the Main theorem. Using this description we complete the proof of the Main theorem in Sect. 6. The Sect. 4 is an independent section devoted to a discussion on regular unipotent elements.

\section{Finite volume, ergodicity and Zariski density}

Let $G$ be a connected Lie group, $\Gamma$ be a lattice in $G$ and $L$ be a subgroup such that the unipotent one-parameter subgroups of $G$ contained in $L$ generate. $L$. Let $X=G / \Gamma$. In this section we note some properties related to closed orbits of the form $F x$, where $x \in X$ and $F$ is a closed subgroup containing $L$ such that $\overline{L x}=F x$.

(2.1) Notation. For $x \in X$ and a subgroup $F \subset G$ define

$$
F_{x}=\{g \in F \mid g x=x\} \text {. }
$$

We first note the following.

(2.2) Lemma. Let $F$ and $H$ be Lie subgroups of $G, Z_{1}$ and $Z_{2}$ be closed orbits of $F$ and $H$, respectively in $X$ and let $Z=Z_{1} \cap Z_{2}$. Then every orbit of $F \cap H$ in $Z$ is both open and closed in $Z$. In particular, for any $x \in X$ there exists a unique smallest Lie subgroup $F$ such that $L C F$ and $F x$ is closed.

Proof. Let $z \in Z$. Then $F z=Z_{1}$ and $H z=Z_{2}$ are closed. Therefore, $F / F_{z} \simeq F z$ and $H / H_{z} \simeq H z$. Also $G_{z}, F_{z}$, and $H_{z}$ are discrete. Therefore, there exists a neighbourhood $\Omega$ of the identity $e$ in $G$ such that $\Omega \Omega^{-1} \cap G_{z}=\{e\},(F z \cap \Omega z)=(F \cap \Omega) z$ and $(H z \cap \Omega z)=(H \cap \Omega) z$. This implies that $(F z \cap H z \cap \Omega z)=(F \cap H \cap \Omega) z$. Hence $(F \cap H) z$ is open in $F z \cap H z=Z$ for every $z \in Z$. Now $(F \cap H) z$ is closed, because its complement in $Z$ is the union of open $F \cap H$ orbits in $Z$ and $Z$ is closed.

One of our aims in this section is to prove the following: 
(2.3) Theorem. For $x \in X$ let $F$ be the smallest subgroup of $G$ such that $L \subset F$ and $F x$ is closed. Then

(a) $F_{x}$ is a lattice in $F$ and

(b) Lacts ergodically on $F x$ with respect to the $F$-invariant probability measure. In particular, $F x$ contains a dense L-orbit.

We recall some preliminaries and a result due to Margulis before going to the proof of the theorem.

(2.4) Definition. A subgroup $H$ of $G$ is said to have property $(D)$ if for every locally finite $H$-invariant measure $\sigma$ on $X$, there exist measurable $H$-invariant subsets $X_{i}$, $i \in \mathbb{N}$ such that $\sigma\left(X_{i}\right)<\infty$ for all $i \in \mathbb{N}$ and $X=\bigcup_{i \in \mathbb{N}} X_{i}$.

In particular, if $H$ has property $(D)$ then every locally finite ergodic $H$-invariant measure on $X$ is finite.

(2.5) Proposition [5, Theorem 4.3]. Any unipotent subgroup UCG has property $(D)$.

(2.6) Definition. Let $F$ be a topological group, $H \subset F$ and $L C F$. We say that the triple $(F, H, L)$ has the Mautner property if the following condition is satisfied: for any continuous unitary representation of $F$ on a Hilbert space $\mathscr{H}$, if a vector $\xi \in \mathscr{H}$ is fixed by $L$ then it is also fixed by $H$.

The following Proposition is a slight modification of Theorem 1.1 in [16].

(2.7) Proposition. Let $F$ be a Lie group and $L$ be a subgroup such that the unipotent one-parameter subgroups contained in $L$ generate $L$. Then there exists a closed normal subgroup $H$ of $F$ such that (i) $L \subset H$ and (ii) the triple $(F, H, L)$ has the Mautner property.

Proof. Let $U$ be a unipotent one-parameter subgroup contained in $L$. By Theorem 1.1 of [16], there exists a normal subgroup $H_{U} \subset F$ such that (a) $\left(F, H_{U}, U\right)$ has the Mautner property and (b) the image of $\operatorname{Ad}(U)$ in the automorphism group of the Lie algebra of $F / H_{U}$ is relatively compact. For each $u \in U$, $\operatorname{Ad} u$ is a unipotent transformation of the Lie algebra of $F$, therefore the image of $U$ in $F / H_{U}$ is in the center. Hence the group $U H_{U}$ is normal in $F$ and $\left(F, U H_{U}, U\right)$ has the Mautner property.

Suppose unipotent one-parameter subgroups $U_{1}, \ldots, U_{n}$ generate $L$. Let $H_{1}, \ldots, H_{n}$ be normal subgroups of $F$ such that $U_{i} \subset H_{i}$ and the triples $\left(F, H_{i}, U_{i}\right)$ have the Mautner property for all $1 \leqq i \leqq n$. Then $H=\bar{H}_{1} \ldots H_{n}$ satisfies the conditions (i) and (ii).

The proof of Theorem 2.3 depends on the following observation by Margulis.

(2.8) Lemma [14, Remarks 3.12]. Suppose $H \subset G$ admits a Levi decomposition $H=S \cdot N$, where $S$ is a semisimple group without compact factors and $N$ is the unipotent radical of $H$. Then $H$ has property $(D)$.

Proof. Let $\sigma$ be a locally finite $H$-invariant measure on $X$. $H$ admits a left regular unitary representation on $\mathscr{L}^{2}(X, \sigma)$.

Let $W$ be a maximal unipotent subgroup of $S$. Then $W \cdot N$ is a unipotent subgroup of $G$. By Proposition 2.5 there exists a measurable $W \cdot N$ invariant partition $\left\{X_{i}\right\}_{i \in \mathbf{N}}$ of $X$ such that $\sigma\left(X_{i}\right)<\infty$ for all $i \in \mathbb{N}$. If $\chi_{i}$ denotes the characteristic function of $X_{i}$ then $\chi_{i}$ is a $W \cdot N$ invariant function in $\mathscr{L}^{2}(X, \sigma)$. By 
Proposition 2.7 there exists a normal subgroup $Q$ of $G$ containing $W \cdot N$ such that $\chi_{i}$ is $Q$ invariant for all $i \in \mathbb{N}$. Since $S$ is a semisimple group without compact factors, $S \subset Q$. Hence $X_{i}$ is $H$ invariant for all $i \in \mathbb{N}$. This completes the proof.

Now we describe the group theoretic structure of a closed subgroup generated by unipotent one-parameter subgroups.

(2.9) Lemma. Let $H \subset G$ be a closed subgroup which is generated by unipotent oneparameter subgroups contained in it. Then $H$ admits a Levi decomposition $H=S \cdot N$, where $S$ is a semisimple group with no compact factors and $N$ is the unipotent radical of $H$.

Proof. It is enough to prove the lemma for the adjoint group of $G$. Therefore we may assume that $G \subset G L(n, \mathbb{R})$ and its unipotent elements are unipotent linear transformations. By Levi decomposition $H=S \cdot R$, where $S$ is a connected semisimple group and $R$ is the radical of $H$ (cf. [18, Sect. P.1.3]). Suppose $H_{1}$ is a normal subgroup of $H$ containing $R$ such that $H / H_{1}$ is a compact semisimple group. Note that under a surjective morphism a unipotent element projects to a unipotent element. Since compact semisimple groups contain no nontrivial unipotent elements, by hypothesis $H=H_{1}$. This shows that $S$ has no compact factors.

To prove the other part we argue as follows; we refer the reader to [18, Preliminaries 2] for the results used in the argument.

Let $\mathbf{H}$ be the smallest algebraic $\mathbb{R}$-subgroup of $G L(n, \mathbb{C})$ containing $H$. Let $\mathbf{N}$ be the unipotent radical of $\mathbf{H}$. By Levi decomposition there exists a connected semisimple $\mathbb{R}$-subgroup $\mathbf{S} \subset \mathbf{H}$ such that $\mathbf{S} \cdot \mathbf{N}$ is a normal subgroup of $\mathbf{H}$ and $\mathbf{T}=\mathbf{H} /(\mathbf{S} \cdot \mathbf{N})$ is an algebraic $\mathbb{R}$-torus. Now the projection of any unipotent element of $\mathbf{H}$ in $\mathbf{T}$ is unipotent. But any algebraic torus contains only semisimple elements. Hence by hypothesis $H \subset \mathbf{S} \cdot \mathbf{N}$. By minimality of $\mathbf{H}, \mathbf{H}=\mathbf{S} \cdot \mathbf{N}$.

Since $H$ normalizes the Lie subalgebra $\mathrm{r}$ corresponding to its radical $R$, by definition $\mathbf{H}$ normalizes $\mathbf{r} \otimes \mathbb{C}$. Hence $R$ is contained in the radical of $\mathbf{H}$. Since the radical of $\mathbf{H}$ is unipotent, $R$ consists of unipotent linear transformations. This completes the proof.

We also need the following lemma.

(2.10) Lemma. Let $F$ be a Lie group, $A$ be a discrete subgroup of $F$ and $H$ be $a$ normal subgroup of $F$ such that $\overline{H A}=F$. Then $H$ acts ergodically on $(F / \Lambda, \sigma)$, where $\sigma$ is a locally finite $F$-semi-invariant measure on $F / A$ with the modular function of $F$ as its character (cf. [18, Sect. 1.4]).

Proof. The proof of Lemma 8.2 in [2] goes through as it is, if we replace $\mathscr{L}^{2}(F / \Lambda, \sigma)$ by the spce of locally integrable functions on $(F / \Lambda, \sigma)$.

Proof of Theorem 2.3. By Proposition 2.7 there exists a smallest closed normal subgroup $H$ of $F$ containing $L$ such that the triple $(F, H, L)$ has the Mautner property.

Since $H$ is normal in $F, H F_{x}$ is a subgroup of $F$. If $H_{1}=\overline{H F_{x}}$ then $H_{1} \supset H$ and $H_{1} x$ is closed in $F x$. By minimality of $F, H_{1}=F$. Hence $\widetilde{H F_{x}}=F$.

Let $H^{\prime}$ be the closure of the group generated by all unipotent one-parameter subgroups of $G$ contained in $H$. Then $L \subset H^{\prime}$ and $H^{\prime}$ is normal in $F$. Therefore, by the hypothesis on $H, H^{\prime}=H$.

Let $\sigma$ be a locally finite $F$-semi-invariant measure on $F / F_{x}$ with a character $\Delta_{F}$, where $\Delta_{F}$ is a modular function of $F$. If $\mathrm{f}$ is the Lie subalgebra corresponding to $F$ then $\Delta_{F}(f)=\left|\operatorname{det}\left(\left.\operatorname{Ad} f\right|_{\mathrm{r}}\right)\right|$ for all $f \in F$. 
Since $H$ is the closure of a subgroup generated by unipotent one-parameter subgroups, $\Delta_{F}(H)=1$. Therefore, $\sigma$ is $H$-invariant. By Lemma $2.10, H$ acts ergodically on $\left(F / F_{x}, \sigma\right)$. Since $F x$ is closed, the natural inclusion $F / F_{x} \hookrightarrow X$ is proper. Therefore, we may treat $\sigma$ as a locally finite ergodic invariant measure of $H$ on $X$. By Lemmas 2.8 and $2.9, H$ has property $(D)$. Hence $\sigma$ is finite. But a finite $F$-semi-invariant measure $\sigma$ must be $F$-invariant. Now by the Mautner property of the triple $(F, H, L), L$ also acts ergodically on $(F x, \sigma)$.

Our next aim is to show that $F$ as in Theorem 2.3 is contained in the Zariski closure of $F_{x}$ (cf. Corollary 2.13). We first prove the following result related to Borel's density theorem.

(2.11) Proposition. Let $F$ be a connected Lie group, $\Delta \neq\{e\}$ be a discrete subgroup of $F$ and $U=\left\{u_{t}\right\}_{t \in \mathbb{R}}$ be a one-parameter subgroup of $F$ such that $\overline{U \Delta}=F$. Let $\varrho: F \rightarrow G L(E)$ be a finite dimensional representation of $F$ such that $\varrho(U)$ consists of unipotent linear transformations of $E$. Then every $\Delta$-stable subspace of $E$ is also F-stable.

Proof (cf. [9, Proposition 9]). Let $W$ be $\Delta$-stable subspace of $E$. Passing to a suitable exterior power of $\varrho$, we may assume that $\operatorname{dim}(W)=1$. Let $\varrho: F \rightarrow P G L(E)$ be the projective linear representation of $F$ on the projective space $\mathbb{P}^{1}(E)$. Let $w \in W \backslash\{0\}$ and $\varphi: \mathbb{R} \rightarrow E$ be the map given by $\varphi(t)=\varrho\left(u_{t}\right) w$ for all $t \in \mathbb{R}$. Fix an orthonormal basis $\left\{e_{1}, \ldots, e_{n}\right\}$ of $E$ with respect to some inner product. Since $\varrho(U)$ consists of unipotent linear transformations, there exist polynomials $\varphi_{1}, \ldots, \varphi_{n}$ on $\mathbb{R}$ such that $\varphi(t)=\sum_{i=1}^{n} \varphi_{i}(t) e_{n}$ for all $t \in \mathbb{R}$. Now $\varphi_{i}^{2}(t) / \sum_{j=1}^{n} \varphi_{j}^{2}(t)$ converges as $t \rightarrow \infty$ for $1 \leqq i \leqq n$. Hence $\lim _{t \rightarrow \infty} \varphi(t) /\|\varphi(t)\|=p$ for some $p \in E \backslash\{0\}$.

If $\operatorname{dim} F=1$ then $U=F$ and there exists $t_{0} \in \mathbb{R} \backslash\{0\}$ such that $u_{k t_{0}} \in \Delta$ for all $k \in \mathbb{N}$. Therefore, $\varrho(\Delta) w=w$ and $\varphi\left(k t_{0}\right)=w$ for all $k \in \mathbb{N}$. Since $\varphi$ is a polynomial function, it must be constant. Thus $\varrho(F) w=w$ in this case.

Suppose $\operatorname{dim} F>1$. Since $\overline{U \Delta}=F$, for every $f \in F$, there exist sequences $\left\{t_{k}\right\}_{k \in \mathbb{N}} \subset \mathbb{R}$, and $\left\{\delta_{k}\right\}_{k \in \mathbb{N}} \subset \Delta$ such that $t_{k} \rightarrow \infty$ and $u_{t_{k}} \delta_{k} \rightarrow f$ as $k \rightarrow \infty$. For $x \in E \backslash\{0\}$, let $\bar{x}$ denote its image in $\mathbb{P}^{1}(E)$. Since $\bar{\varrho}(\Delta) \bar{w}=\bar{w}$,

$$
\bar{\varrho}(f) \bar{w}=\lim _{k \rightarrow \infty} \bar{\varrho}\left(u_{t_{k}} \delta_{k}\right) \bar{w}=\lim _{k \rightarrow \infty} \bar{\varrho}\left(u_{t_{k}}\right) \bar{w}=\lim _{k \rightarrow \infty} \overline{\varphi\left(t_{k}\right)}=\bar{p} .
$$

Putting $f=$ identity we get $\bar{p}=\bar{w}$. Hence $\bar{\varrho}(F) \bar{w}=\bar{w}$.

(2.12) Corollary. Let $F$ be a connected Lie subgroup of $G$ and $L$ be a subgroup of $F$ generated by unipotent one-parameter subgroups $U_{1}, \ldots, U_{k}$ of $G$. Suppose $L x=F x$. Let $\varrho: F \rightarrow G L(E)$ be a finite dimensional representation such that for each $1 \leqq i \leqq k$, $\varrho\left(U_{i}\right)$ consists of unipotent linear transformations of $E$. Then every $F_{x}$-stable subspace of $E$ is $F$-stable.

Proof. Fix $i$. Let $F_{i}$ be the smallest closed subgroup of $F$ such that $F_{i} \supset U_{i}$ and $F_{i} x$ is closed. By Theorem 2.3 there exists $g_{i} \in F_{i}$ such that $\overline{U_{i} g_{i} x}=F_{i} g_{i} x=F_{i} x$. Let $\Delta=F_{x}$ and $\Delta_{i}=\Delta \cap F_{i}$. Then $\overline{U_{i} g_{i} \Delta_{i} g_{i}^{-1}}=F_{i}$.

Now let $W$ be a $\Delta$-stable subspace of $E$. Let $W_{i}=\varrho\left(g_{i}\right) W$. Then $W_{i}$ is stabilized by $\left(g_{i} A_{i} g_{i}^{-1}\right)$. By Proposition 2.11, $W_{i}$ is stabilized by $F_{i}$. Since $g_{i}^{-1} \in F_{i}$, we have $W_{i}=\varrho\left(g_{i}^{-1}\right) W_{i}=W$. Therefore, $F_{i}$ stabilizes $W$. This happens for each $i=1, \ldots, k$. Therefore, $\varrho(L) W=W$ and hence $\varrho(L \Delta) W=W$. Now by continuity of $\varrho$ we have $Q(F) W=W$.

(2.13) Corollary. Let the notation be as above. Suppose further that $G$ is the component of identity in $\mathbf{G}_{\mathbf{R}}=\mathbf{G} \cap G L(n, \mathbb{R})$, where $\mathbf{G} \subset G L(n, \mathbb{C})$ is an algebraic 
subgroup defined over $\mathbb{R}$. Let $F$ be a connected Lie subgroup of $G$ and $L$ be a subgroup generated by all algebraic unipotent one-parameter subgroups contained in $F$. Suppose $x \in X$ is such that $\overline{L x}=F x$. Then $F$ is contained in the Zariski closure of $F_{x}$ in $G L(n, \mathbb{R})$.

Proof. Let $P_{d}$ be the space of real polynomials of degree $\leqq d$ defined on $M(n, \mathbb{R})$, the space of $n \times n$ matrices with real entries. Consider the representation $\varrho$ of $G L(n, \mathbb{R})$ on $P_{d}$ defined as follows: for $g \in G, p \in P_{d}$ and $x \in M(n, \mathbb{R}),[\varrho(g) p](x)$ $=p\left(g^{-1} x\right)$. Clearly, $\varrho(g) p \in P_{d}$. Since $\varrho: G L(n, \mathbb{R}) \rightarrow G L\left(P_{d}\right)$ is an algebraic morphism, $\varrho$ preserves algebraic unipotent subgroups. Thus $\varrho$ restricted to $F$ satisfies the conditions of Corollary 2.12. Define $I_{d}=\left\{p \in P_{d} \mid p(\delta)=0\right.$ for all $\left.\delta \in F_{x}\right\}$. Since $F_{x}$ is a group, $F_{x}$ stabilizes $I_{d}$. Therefore, by Corollary 2.12, for all $f \in F$ and $p \in I_{d}$ we have $\varrho\left(f^{-1}\right) p \in I_{d}$ and hence $p(f)=\left[\varrho\left(f^{-1}\right) p\right](e)=0$. Thus $p(f)=0$ for all $f \in F, p \in I_{d}$ and $d \geqq 0$. This shows that $F$ is in the Zariski closure of $F_{x}$ in $G L(n, \mathbb{R})$.

\section{On subgroups with closed orbits}

(3.1) Proposition. Let $G$ be a connected semisimple Lie group without compact factors, with trivial center and of $\mathbb{R}$-rank $=1$. Let $\Gamma$ be a lattice in $G$ and $L \neq\{e\}$ be a subgroup generated by unipotent elements of $G$ contained in $L$. Let $x \in G / \Gamma$ and suppose that $\overline{L x}=F x$ for a connected Lie subgroup $F \subset G$. Then either

(a) $F$ is a reductive group with compact center, or

(b) $F$ is a unipotent subgroup of $G$.

Proof. $G=\mathbf{G}_{\mathbf{R}}^{0}$ for a semisimple algebraic $\mathbb{R}$-group $\mathbf{G}$ (cf. [21, Sect. 3.1.6]). Let $\mathbf{F}$ be the smallest algebraic $\mathbb{R}$-subgroup of $\mathbf{G}$ containing $F$.

Suppose that the unipotent radical $\mathbf{R}_{u}(\mathbf{F})$ is trivial. Then $\mathbf{F}_{\mathbf{R}}^{0}$ is a reductive group (cf. [18, Prelim. 2.5]). Since the $\mathbb{R}$-rank of $G$ is 1 and the commutator subgroup of $\mathbf{F}_{\mathbf{R}}^{0}$ is noncompact, this also implies that the center of $\mathbf{F}_{R}^{0}$ is compact. Since $F$ is Zariski dense in $\mathbf{F}$, the radical of $F$ is contained in the radical of $F_{R}^{0}$. Hence $F$ is a reductive group with compact center.

Suppose the unipotent radical of $\mathbf{F}_{\mathbf{R}}^{0}$ is nontrivial. Let $N$ be a maximal unipotent subgroup of $G$ containing $\mathrm{R}_{\mathrm{u}}\left(\mathbf{F}_{\mathrm{R}}^{0}\right)$. Then $F \subset P=\mathrm{N}_{\mathrm{G}}(N)$, the normalizer of $N$ in $G$ (cf. [18, Sect. 12.6]). Since $N$ contains all unipotent elements in $P, L \subset N$. If $F=\bar{L}$ then we are through. Otherwise, since $\overline{L x}=F x$, there exist sequences $\left\{l_{i}\right\} \subset L$ and $\left\{f_{i}\right\} \subset F$ such that $l_{i} \rightarrow \infty, f_{i} \rightarrow e$ and $l_{i} x=f_{i} x$ for all $i \in \mathbb{N}$. Now for all large $i, \gamma_{i}$ $=f_{i}^{-1} l_{i} \in F_{x} \backslash\{e\}$. Since $P$ is a minimal parabolic subgroup of $G, P$ admits a decomposition $P=Z_{\mathrm{G}}(A) \cdot N$, where $A$ is a Cartan subgroup of $G$ and $\mathrm{Z}_{\mathrm{G}}(A)$ is the centralizer of $A$ in $G$. There exists $Y$ in the Lie algebra of $A$ such that if $q=\exp (-Y)$ then for all $l \in N, q^{n} l q^{-n} \rightarrow e$ as $n \rightarrow \infty$. Therefore there exists an increasing sequence $\left\{n_{i}\right\}_{i \in \mathbf{N}}$ such that $q^{n_{i}} \gamma_{i} q^{-n_{i}} \rightarrow e$ as $i \rightarrow \infty$. For each $j \in \mathbb{N}$, put $\gamma_{j}^{\prime}=e$ if $j<n_{1}$, and put $\gamma_{j}^{\prime}=\gamma_{n_{1}}$ if $n_{i} \leqq j<n_{i+1}$. Then $q^{n} \gamma_{n}^{\prime} q^{-n} \rightarrow e$ as $n \rightarrow \infty$. Therefore by Lemmas 3.5 and 3.6 in [12], $N \cap G_{x} \neq \emptyset$. Now by Lemmas 3.16 and 3.17 in [12], $N / N \cap G_{x}$ is compact, equivalently $N x$ is compact. This shows that $\overline{L x} \subset N x$. Hence $F \subset N$. This completes the proof.

In view of arithmeticity of irreducible lattices in semisimple groups of rank greater than 1, we formulate the next result in the setup of algebraic groups.

(3.2) Proposition. Let $G=\mathbf{G}_{\mathbf{R}}^{0}$ for an algebraic $\mathbf{Q}$-group $\mathbf{G} \subset S L(n, \mathbb{C}), \Gamma=G$ $\cap S L(n, \mathbb{Z})$ and $L$ be a subgroup generated by algebraic unipotent one-parameter subgroups of $G$ contained in $L$. Suppose $\overline{L \bar{\Gamma}}=F \Gamma$ for a connected Lie subgroup $F$ of 
G. Let $\mathbf{F}$ be the smallest algebraic $\mathbf{Q}$-group containing $L$. Then the radical of $\mathbf{F}$ is a unipotent algebraic $\mathbb{Q}$-group and $F=\mathbf{F}_{\mathbf{R}}^{\mathbf{0}}$.

Proof. By the arguments as in Lemma 2.9, it follows that the radical of $\mathbf{F}$ is a unipotent algebraic $\mathbb{Q}$-group. Therefore $\mathbf{F}$ admits no nontrivial character defined over $\mathbb{Q}$. Let $A=S L(n, \mathbb{Z})$ and $\Delta=\mathrm{F}_{\mathrm{R}}^{0} \cap A$. Then the natural inclusion map $\mathrm{i}: \mathrm{F}_{\mathbb{R}}^{0} / \Delta$ $\rightarrow S L(n, \mathbb{R}) / \Lambda$ is proper (cf. [18, Sect. 10.15]). Let $\mathbf{j}: G / \Gamma \rightarrow S L(n, \mathbb{R}) / \Lambda$ be the natural inclusion map. Now $F_{R}^{0} \Gamma=j^{-1}\left(F_{R}^{0} \Lambda\right)$ is closed in $G$. Therefore $F \subset F_{R}^{0}$. Hence $\overline{L A}$ $=F \Delta$ in $F_{R}^{0} / \Delta$. Since $i$ is proper, $L A=F \Lambda$.

Note that $\Lambda$ is a lattice in $S L(n, \mathbb{R})$ (cf. [18, Sect. 10.5]). Let $\mathbf{F}^{\prime}$ be the Zariski closure of $\Delta$ in $\mathbf{G}$. Therefore $\mathbf{F}^{\prime}$ is defined over $\mathbb{Q}$ (cf. [21, Sect. 3.1.8]). By Corollary $2.13, F \subset F^{\prime}$. Since $L \subset F$, by minimality of $F$ we have $F^{\prime}=F$. Hence $F$ is Zariski dense in $\mathbf{F}$.

By Levi decomposition $F=S \cdot R$, where $S$ is a semisimple group and $R$ is the radical of $F$. Let $\mathbf{S}$ and $\mathbf{R}$ be the smallest algebraic $\mathbb{R}$-groups containing $S$ and $R$, respectively. Now $\mathbf{S}$ normalizes $\mathbf{R}$ and $\mathbf{R}$ is a solvable group. Therefore $\mathbf{F}=\mathbf{S} \cdot \mathbf{R}$. Since the radical of $\mathbf{F}$ is unipotent, $\mathbf{S}$ is a semisimple group and $\mathbf{R}$ is a unipotent group. Therefore $R=\mathbf{R}_{\mathbf{R}}^{0}$ (cf. [18, Sect. P.2.2]). Now $S$ is a connected normal Zariski dense subgroup of the semisimple group $\mathbf{S}_{\mathbf{R}}^{0}$. Hence $S=\mathbf{S}_{\mathbf{R}}^{0}$. Thus $F=\mathbf{S}_{\mathbf{R}}^{\mathbf{0}} \cdot \mathbf{R}_{\mathbf{R}}^{\mathbf{0}}=\mathbf{F}_{\mathbf{R}}^{\mathbf{0}}$.

(3.3) Notation. For a semisimple Lie group $G$ and a parabolic subgroup $P$, define ${ }^{0} P=\left\{g \in P \mid \operatorname{det}\left(\left.\operatorname{Ad} g\right|_{w}\right)=1\right\}$, where $w$ is the Lie subalgebra corresponding to the unipotent radical of $P$.

(3.4) Lemma. Let $G=\mathbf{G}_{\mathbf{R}}^{0}$ for a semisimple algebraic $\mathbb{Q}$-group $\mathbf{G}, \Gamma=G \cap S L(n, \mathbb{Z})$ and $U=\mathbf{U}_{\mathbf{R}}^{0}$ for a unipotent algebraic $\mathbb{Q}$-subgroup $\mathbf{U} \subset \mathbf{G}$. Then there exists a parabolic $\mathbf{Q}$-subgroup $\mathbf{P} \subset \mathbf{G}$ such that if $\boldsymbol{P}=\mathbf{P}_{\mathbf{R}}^{0}$ and $W=\mathbf{R}_{\mathrm{u}}(P)$ then (a) $U \subset W$ and $\mathrm{N}_{\mathrm{G}}(U) \subset P$, (b) $W / W \cap \Gamma$ is compact and (c) ${ }^{\circ} P \Gamma$ is closed.

Proof. Let $\mathbf{P}$ be the maximal algebraic $\mathbb{Q}$-subgroup of $\mathbf{G}$ such that $U \subset \mathbf{R}_{\mathrm{u}}(\mathbf{P})$ and $\mathbf{N}_{G}(U) \subset \mathbf{P}$. Let $\mathbf{W}=\mathbf{R}_{\mathrm{u}}(\mathbf{P})$. Then $\mathbf{W}$ is a $\mathbb{Q}$-group, $\mathbf{P}_{1}=\mathbf{N}_{\mathbf{G}}(\mathbf{W})$ is a $\mathbb{Q}$-group, $\mathbf{P} \subset \mathbf{P}_{\mathbf{1}}$ and $\mathbf{W} \subset \mathbf{R}_{u}\left(\mathbf{P}_{1}\right)$. By the maximality $\mathbf{P}_{1}=\mathbf{P}$. Therefore $\mathbf{P}$ is a parabolic subgroup of $\mathbf{G}$ (cf. [18, Sect. 12.8]). This proves (a).

Let $W=\mathbf{W}_{\mathbf{R}}^{0}$. Since $\mathbf{W}$ is defined over $\mathbb{Q}, W / W \cap \Gamma$ is compact (cf. [18, Sect. 2.13]).

Let $g$ and $\mathbf{w}$ be Lie algebras corresponding to $G$ and $W$, respectively. Let $r=\operatorname{dim}(w)$ and $\varrho: G \rightarrow G L\left(\wedge^{r} \mathbf{g}\right)$ be the $r$-th exterior of the Adjoint representation Ad: $G \rightarrow G L(g)$. Let $\left\{e_{1}, \ldots, e_{r}\right\}$ be a basis of $w$ and put $p=e_{1} \wedge \ldots \wedge e_{r}$. Then for all $a \in P, a p=(\operatorname{Ad} a) e_{1} \wedge \ldots \wedge(\operatorname{Ad} a) e_{r}=\left(\left.\operatorname{det} \operatorname{Ad} a\right|_{w}\right) p$. Therefore, ${ }^{\circ} P=\{g \in G \mid \varrho(g) p=p\}$.

Since $\mathbf{G}$ and $\mathbf{W}$ are defined over $\mathbf{Q}, \mathbf{g}$ and $\mathbf{w}$ admit compatible rational structures. Now $\wedge^{\prime} \mathbf{g}, \wedge^{r} \mathbf{w}$ and $\varrho$ are defined over $\mathbb{Q}$. Fix a rational basis of $\wedge^{r} \mathbf{g}$. For a nonzero rational vector $p \in \Lambda^{r} \mathrm{w}$, let $\alpha: G \rightarrow \Lambda^{r} \mathrm{~g}$ be the map defined by $\alpha(g)=\varrho(g) p$ for all $g \in G$. Then $\alpha$ is defined over $\mathbb{Q}$. Therefore $\alpha(\Gamma)$ consists of rational points with bounded denominators in each co-ordinate. Hence $\alpha(\Gamma)$ is discrete and $\Gamma^{0} P$ $=\alpha^{-1}(\alpha(\Gamma))$ is closed. Therefore ${ }^{0} P \Gamma$ is closed.

The next lemma is useful when we want to change a lattice by a commensurable one.

(3.5) Lemma. Let $G$ be a Lie group and $\Gamma$ and $\Gamma^{\prime}$ be discrete subgroups of $G$. Suppose $\Gamma^{\prime} \subset \Gamma$ and $\left[\Gamma: \Gamma^{\prime}\right]<\infty$. Let $\varphi: X^{\prime}=G / \Gamma^{\prime} \rightarrow X=G / \Gamma$ be the natural quotient map. Let $x^{\prime} \in X^{\prime}, x=\varphi\left(x^{\prime}\right) \in X$ and $F$ and $H$ be closed connected subgroups $G$. Then the 
following statements hold.

1. $\overline{F x^{\prime}}=H x^{\prime} \Rightarrow \overline{F x}=H x$.

2. $\overline{F x}=H x \Rightarrow H x^{\prime}$ is closed and $\overline{F x^{\prime}}$ contains an open subset of $H x^{\prime}$.

3. If $\Gamma$ is a lattice in $G$ and $F$ is generated by unipotent one-parameter subgroups of $G$ then $\overline{F x}=H x \Rightarrow \overline{F x^{\prime}}=H x^{\prime}$.

Proof. Since $\left[\Gamma: \Gamma^{\prime}\right]=n<\infty$, the map $\varphi$ is proper. If $\overline{F x^{\prime}}=H x^{\prime}$ then by properness of $\left.\varphi, \overline{F x}=\overline{\varphi\left(F x^{\prime}\right.}\right)=\varphi\left(\overline{F x^{\prime}}\right)=\varphi\left(H x^{\prime}\right)=H s$. This proves 1$)$.

Let $\Gamma^{\prime \prime}=\bigcap_{\gamma \in \Gamma} \gamma \Gamma^{\prime} \gamma^{-1}$. Then $\Gamma^{\prime \prime}$ is a normal subgroup of finite index in $\Gamma$. Therefore in view of 1), we may assume that $\Gamma^{\prime}$ is normal in $\Gamma$ for proving 2) and 3). Therefore $\Gamma$ acts on $X^{\prime}$ from the right and $X=X^{\prime} / \Gamma$.

Suppose $\overline{F x}=H x$. Let $Z^{\prime}=\varphi^{-1}(H x)$ and $E^{\prime}=\overline{F x^{\prime}} \subset Z^{\prime}$. Now $\varphi\left(E^{\prime}\right)=\overline{F x}=H x$ $=\varphi\left(H x^{\prime}\right)$. Hence there exits $\left\{\gamma_{1}, \ldots, \gamma_{n}\right\} \subset \Gamma$ such that $\bigcup_{i=1}^{n} H x^{\prime} \gamma_{i}=Z^{\prime}=\bigcup_{i=1}^{n} E^{\prime} \gamma_{i}$. This shows that $H x^{\prime}$ and $E^{\prime}$ contain open subsets of $Z^{\prime}$. Therefore $H x^{\prime} \gamma_{i}$ is open in $Z^{\prime}$ for each $i$ and hence $H x^{\prime}$ is closed in $Z^{\prime}$. This proves 2).

Assume the hypothesis in 3). Then $\Gamma^{\prime}$ is also a lattice in $G$. Now since $\overline{F x^{\prime}}$ contains an open subset of $H x^{\prime}, H$ is the smallest Lie subgroup of $G$ such that $F \subset H$ and $H x^{\prime}$ is closed. By Theorem $2.3, H x^{\prime}$ contains a dense orbit of $F$, which must intersect $\overline{F x^{\prime}}$. Therefore $\overline{F x^{\prime}}=H x^{\prime}$. This proves 3 ).

(3.6) Definition. Let $G$ be a Lie group. We call discrete subgroups $\Gamma$ and $\Gamma^{\prime}$ of $G$ commensurable if $\left[\Gamma: \Gamma \cap \Gamma^{\prime}\right]<\infty$ and $\left[\Gamma^{\prime}: \Gamma \cap \Gamma^{\prime}\right]<\infty$.

(3.7) Remark. The Proposition 3.2 and Lemma 3.4 hold when $\Gamma$ is commensurable with $G \cap S L(n, \mathbb{Z})$. To show this use Lemma 3.5 and Theorem 2.3.

The next lemma is useful when we want to factor agroup a group by a compact normal subgroup.

(3.8) Lemma. Let $\varrho: G \rightarrow G_{1}$ be a surjective homomorphism of Lie groups $G$ and $G_{1}$. Let $\Gamma$ be a discrete subgroup of $G$. Suppose $\Gamma_{1}=\varrho(\Gamma)$ is a lattice in $G_{1}$ and the canonical quotient map $\bar{\varrho}: X=G / \Gamma \rightarrow X_{1}=G_{1} / \Gamma_{1}$ is proper. Let $L$ be a subgroup of $G$ generated by unipotent one-parameter subgroups contained in $L$. Suppose $\operatorname{ker}(\varrho)$ normalizes $L$. Let $L_{1}=\varrho(L), x \in X$ and $x_{1}=\bar{\varrho}(x) \in X_{1}$. Then $\overline{L x}=F x$ for a connected Lie subgroup $F$ of $G$ if and only if $L_{1} x_{1}=F_{1} x_{1}$ for a connected Lie subgroup $F_{1}$ of $G_{1}$. In this case $F_{1}=\varrho(F)$.

Proof. Since $\bar{\varrho}$ is proper, $r$ must be a lattice in $G$.

If $\overline{L x}=F x$ then by properness of $\varrho, \overline{L_{1} x_{1}}=\bar{\varrho}(L x)=\varrho(L x)=\bar{\varrho}(\overline{F x})=\varrho(F) x_{1}$.

Suppose $\bar{L}_{1} x_{1}=F_{1} x_{1}$. If $H=\varrho^{-1}\left(F_{1}\right)$ then $H x=\bar{Q}^{-1}\left(F_{1} x_{1}\right)$ is closed. Let $F$ be the smallest Lie subgroup of $H$ such that $F \supset L$ and $F x$ is closed. Now $F_{1} x_{1}$ $=\bar{\varrho}(H x) \supset \bar{\varrho}(F x) \supset \bar{\varrho}(L x)=\bar{L}_{1} x_{1}=F_{1} x_{1}$. Therefore since $F$ and $F_{1}$ are connected, we have $F_{1}=\varrho(F)$. By Theorem 2.3 there exists $y \in F x$ such that $L x=F x$. Since $\bar{\varrho}(\overline{L x})=\bar{\varrho}(F x)$ there exists $h \in \operatorname{ker} \varrho$ such that $h y \in \overline{L x}$. Since $\operatorname{ker}(\varrho)$ normalizes $L, \overline{L x}$ $\supset \overline{L h y}=h \overline{L y}=h F x=\left(h F h^{-1}\right) h y$. Therefore $h F h^{-1} C F$ and by dimension consideration $h F h^{-1}=F$. Hence $L x=F x$.

(3.9) Lemma. Let $G$ be a connected semisimple Lie group without compact factors, with trivial center and of $\mathbb{R}$-rank $>1$. Let $\Gamma$ be an irreducible lattice in $G$ and $L$ be $a$ subgroup generated by unipotent one-parameter subgroups of $G$ contained in $L$. Suppose $\bar{L}=F \Gamma$ for a connected Lie group $F \subset G$. Then the radical of $F$ is $a$ unipotent subgroup of $G$. Moreover, if the radical of $F$ is nontrivial then there exists $a$ proper parabolic subgroup $P$ of $G$ such that (a) $F C^{0} P$, (b) ${ }^{\circ} P \Gamma$ is closed in $G / \Gamma$, and (c) $W / W \cap \Gamma$ is compact, where $W=\mathrm{R}_{\mathrm{u}}(P)$. 
Proof. By the arithmeticity theorem of Margulis (cf. [21, Theorem 6.1.2]), $\Gamma$ is an arithmetic lattice in $G$. That is, there exists a semisimple algebraic $Q$-group $H$ and a surjective homomorphism $\varrho: H \rightarrow G$ such that $\operatorname{ker}(\varrho)$ is compact and $\varrho(\Lambda)$ is commensurable with $\Gamma$, where $H=H_{\mathbb{R}}^{0}$ and $A=H \cap S L(n, \mathbb{Z})$. By Lemma 3.5, there no loss of generality in assuming that $\varrho(\Lambda)=\Gamma$.

There is a normal semisimple subgroup $H_{1} \subset H$ such that $\varrho\left(H_{1}\right)=G$ and $\operatorname{ker}(\varrho)$ $\cap H_{1}$ is discrete. Note that $H_{1}$ contains all unipotent one-parameter subgroups of $H$ and $\operatorname{ker}(\varrho)$ commutes with $H_{1}$. There exists a subgroup $L^{\prime} \subset H_{1}$ such that $L^{\prime}$ is generated by algebraic unipotent one-parameter subgroups contained in it and $\varrho\left(L^{\prime}\right)=L$. If $\bar{\varrho}: H / \Lambda \rightarrow G / \Gamma$ is the natural quotient map then $\bar{\varrho}$ is proper. By Lemma 3.8, there exists a subgroup $F^{\prime} \subset H$ such that $\varrho\left(F^{\prime}\right)=F$ and $\bar{L} \Lambda=F^{\prime} \Lambda$.

By Proposition 3.2, the radical of $F^{\prime}$ is $U^{\prime}=\mathbf{U}_{\mathbf{R}}^{\prime 0}$ for a unipotent $Q$-subgroup $\mathbf{U}^{\prime} \subset \mathbf{H}$. Since $\varrho$ is surjective and $\operatorname{ker}(\varrho)$ is compact, the radical of $F$ is also a unipotent subgroup of $G$.

If $U^{\prime}$ is not trivial then by Lemm 3.4 there exists a proper parabolic subgroup $P^{\prime}$ of $H$ such that a) $L^{\prime} \subset N_{H}\left(U^{\prime}\right) \subset P^{\prime}$, b) ${ }^{0} P^{\prime} A$ is closed in $H / A$, and c) $W^{\prime} / W^{\prime} \cap A$ is compact, where $W^{\prime}=R_{\mathrm{u}}\left(P^{\prime}\right)$. Since $L^{\prime}$ is generated by unipotent one-parameter subgroups, $L^{\prime} C^{0} P^{\prime}$ and hence $F^{\prime} C^{0} P^{\prime}$. If $P=\varrho\left(P^{\prime}\right)$ then $P$ is a proper parabolic subgroup of $G$ with the required properties.

(3.10) Proposition. Let $G$ be a connected semisimple Lie group without compact factors and with trivial center. Let $\Gamma$ be a lattice in $G$ and $L$ be a subgroup generated by unipotent one-parameter subgroups contained in $L$. If $x \in G / \Gamma$ and $L x=F x$ for a connected Lie subgroup $F \subset G$ then one of the following possibilities holds.

1. $F$ is a reductive group with compact center.

2. There is a proper parabolic subgroup $P$ of $G$ such that (a) $F C^{0} P$, (b) ${ }^{\circ} P x$ is closed and (c) $\mathrm{R}_{\mathrm{u}}(P) x$ is compact.

Proof. Let $G_{x}=\Lambda$. For $G$ there exists a direct product decomposition $G=G_{1} \ldots G_{n}$ such that $\Lambda_{k}=G_{k} \cap A$ is an irreducible lattice in $G_{k}$ for $1 \leqq k \leqq n$ and $\left[A: \Lambda_{1} \ldots \Lambda_{n}\right]<\infty$ (cf. [18, Sect. 5.22]). By Lemma 3.5, without loss of generality we may assume that $\Lambda=\Lambda_{1} \ldots \Lambda_{n}$. Then $G / \Lambda \simeq G_{1} / \Lambda_{1} \times \ldots \times G_{n} / \Lambda_{n}$. Let $\varphi_{k}: G \rightarrow G_{k}$ be the projection homomorphism of $G$ onto $G_{k}$ and $\bar{\varphi}_{k}: G / \Lambda \rightarrow G_{k} / \Lambda_{k}$ be the natural projection. Let $x_{k}=\bar{\varphi}_{k}(x), L_{k}=\varphi_{k}(L)$ and $F_{k}=\varphi_{k}(F)$. By Theorem 2.3, Fx supports a finite $F$ invariant measure $\sigma$. Now the projection of $\sigma$ on $F_{k} x_{k}$ is a finite $F_{k}$ invariant measure. Therefore $F_{k} \cap A_{k}$ is a lattice in $F_{k}$. Hence $F_{k} x_{k}$ is closed in $G_{k} / A_{k}$ (cf. [18, Sect. 1.13]). Now $\overline{L_{k} x_{k}}=F_{k} x_{k}$.

Let $R$ be the radical of $F$. Then $R_{k}=\varphi_{k}(R)$ is the radical of $F_{k}$. If $F_{k}$ is reductive for all $k$ then by Proposition 3.1 or Lemma 3.9, $R_{k}$ is compact and abelian for all $k$. Hence $R$ is compact and abelian. In this case 1) holds.

Now suppose $F_{k}$ is not reductive for some $k$. Then by Proposition 3.1 or Lemma 3.9, there exists a parabolic subgroup $P_{k} \subseteq G_{k}$ such that (a) $F_{k} \subset{ }^{0} P$, (b) ${ }^{0} P x_{k}$ is closed and (c) $\mathrm{R}_{\mathrm{u}}\left(P_{k}\right) x_{k}$ is compact. If $P=\left(\prod_{j \neq k} G_{j}\right) P_{k}$ then $P$ is a parabolic subgroup of $G,{ }^{0} P=\left(\prod_{j \neq k} G_{j}\right)^{0} P$, and $R_{u}(P)=R_{u}\left(P_{k}\right)$. Now (a) $F \subset{ }^{0} P$, (b) ${ }^{0} P x$ $=\bar{\varphi}_{k}^{-1}\left({ }^{0} P_{k} x_{k}\right)$ is closed and (c) in view of the natural inclusion $G_{k} / \Lambda_{k} \hookrightarrow G / \Lambda, R_{u}(P) x$ $=\mathrm{R}_{\mathrm{u}}\left(P_{k}\right) x_{k}$ is compact.

\section{Regular unipotent elements}

(4.1) Definition. Let $G$ be a connected reductive Lie group. A unipotent element of $G$ is called regular if it is contained in a unique maximal unipotent subgroup of $G$. A 
unipotent one-parameter subgroup of $G$ is called regular if it contains a regular unipotent element.

(4.2) Lemma. Let $\varrho: G \rightarrow G^{\prime}$ be a homomorphism of connected reductive Lie groups. Let $u \in G$ be a unipotent element.

1. If $\varrho$ is surjective and $u$ is regular in $G$ then $\varrho(u)$ is regular unipotent in $G^{\prime}$.

2. If $\operatorname{ker} \varrho \subset Z(G)$ and $\varrho(u)$ is regular unipotent in $G^{\prime}$ the $u$ is regular in $G$.

Proof. 1) follows immediately from the definition.

Suppose $\operatorname{ker} \varrho \subset Z(G)$. Since $G$ is connected and reductive, $G=Z(G) S$, where $S$ is a connected semisimple group. Now any maximal unipotent subgroup of $G$ is of the form $\mathrm{Z}(G) N$, where $N$ is a maximal unipotent subgroup of $S$. Since $S$ is semisimple and connected, $\varrho(N)$ is a unipotent subgroup of $G^{\prime}$ (cf. [21, Sect. 3.4.2]). Now 2) easily follows from the definition.

(4.3) Theorem (cf. [20, Theorem 3.7]). Let $G$ be a connected semisimple Lie group with trivial center, $A$ be a Cartan subgroup of $G$ and $P_{0}=M A N$ be a minimal parabolic subgroup of $G$, where $M$ is a maximal compact connected subgroup of $Z_{\mathrm{G}}(A)$ and $N$ is a maximal unipotent subgroup of $G$. Let $u$ be a unipotent element of $G$. Then the following statements are equivalent.

(a) $u$ is regular.

(b) $u$ belongs to a unique conjugate of a given parabolic subgroup of $G$.

(c) If $u \in N$ and $u=\exp \left(\sum_{\alpha \in R^{+}} X_{\alpha}\right)$ then for every simple root $\alpha, X_{\alpha}+X_{2 \alpha} \neq 0$. Here $R^{+}$is the set of positive roots associated to the parabolic pair $\left(P_{0}, A\right)$ and $X_{\alpha}$ is an element of the $\alpha$-root space of $A$.

Proof. We refer to [18, Chap. 12] for the results used in this proof. First note the following. $G=\mathbf{G}_{\mathbb{R}}^{0}$ for an algebraic $\mathbb{R}$-group $\mathbf{G}$. If $P$ is a parabolic subgroup of $G$ then $P=\mathbf{P} \cap G$. Let $W=\mathbf{R}_{\mathrm{u}}(P)$. There exists a semisimple $\mathbb{R}$-subgroup $\mathbf{S} \subset \mathbf{P}$ such that if $S=\mathbf{S}_{\mathbf{R}}^{0}$ then any unipotent element of $P$ is contained in $S \cdot W$ (cf. [18, Prelim. 2.5]). Therefore any maximal unipotent subgroup of $P$ is of the form $V \cdot W$, where $V$ is a maximal unipotent subgroup of $S$. Since $S$ is a semisimple group, any two maximal unipotent subgroups of $S$ are conjugates. Therefore, any two maximal unipotent subgroups of $P$ are conjugate by an element of $P$. Moreover, any maximal unipotent subgroup of $G$ is conjugate to a subgroup of $P$.

Now assume (a). Let $N^{\prime}$ be a maximal unipotent subgroup of $G$ containing $u$. Suppose there exist $g_{1}, g_{2} \in G$ such that $g_{1} u g_{1}^{-1}, g_{2} u g_{2}^{-1} \in P$. By regularity of $g_{i} u g_{i}^{-1}$ and the observations made above, $g_{i} N^{\prime} g_{i}^{-1} \subset P$ for $i=1,2$. Now there exists $p \in P$ such that $g_{1} N^{\prime} g_{1}^{-1}=p g_{2} N^{\prime} g_{2}^{-1} p^{-1}$. Since the normalizer of a maximal unipotent subgroup of $P$ is contained in $P$, we have $p g_{2} g_{1}^{-1} \in P$. Hence $g_{1}^{-1} P g_{1}=g_{2}^{-1} P g_{2}$. Thus (a) $\Rightarrow(b)$.

Assume the contrary to (c). Suppose $u \in P_{0}, u=\exp \left(\sum_{\alpha \in R^{+}} X_{\alpha}\right)$ and for some simple root $\beta, X_{\beta}+X_{2 \beta}=0$. Let $X_{-\beta} \neq 0$ be an element of the $(-\beta)$-root space. If $\alpha \in R^{+}$and $\alpha \neq \beta$ or $2 \beta$ then for every $k \in \mathbb{N}$, either $\alpha-k \beta \in R^{+}$or $\alpha-k \beta$ is not a root. Since $\operatorname{ad}^{k} X_{-\beta}\left(X_{\alpha}\right)$ belongs to the $(\alpha-k \beta)$-root space, we have $\operatorname{Adg}\left(\sum_{\alpha \in R^{+}} X_{\alpha}\right)=\sum_{\alpha \in R^{+}} Y_{\alpha}$, where $g=\exp \left(X_{-\beta}\right)$ and $Y_{\alpha}$ belongs to the $\alpha$-root space. Therefore, $g u g^{-1} \in P_{0}$. Hence $u \in P_{0} \cap g P_{0} g^{-1}$. But $g \notin P_{0}$. This contradicts (b). Thus (b) $\Rightarrow$ (c).

Let $u \in N$ be such that $u=\exp \left(\sum_{\alpha \in R^{+}} X_{\alpha}\right)$ and $X_{\beta}+X_{2 \beta} \neq 0$ for every simple root $\beta$. Suppose for some $g \in G, u \in g N g^{-1}$. By Bruhat decomposition of $G, g=p_{1} w^{*} p_{2}$ 
where $p_{1}, p_{2} \in P_{0}$ and $w^{*} \in \mathrm{N}_{G}(A)$ represents a Weyl group element $w \in \mathrm{W}(G, A)$. Therefore $g u g^{-1}=\left(p_{2}^{-1} w^{*-1} p_{1}^{-1}\right) u\left(p_{1} w^{*} p_{2}\right) \in N$. Hence $w^{*-1}\left(p_{1}^{-1} u p_{1}\right) w^{*} \in P_{0}$. Now $u^{\prime}=p_{1} u p_{1}^{-1}=\exp \left(\sum_{\alpha \in R^{+}} X_{\alpha}^{\prime}\right) \in P_{0}$. Suppose $p_{1}=a m n$, where $a \in A, m \in M$ and $n \in N$. Then for every simple root $\beta, X_{\beta}^{\prime}=\operatorname{Ad}(a m) X_{\beta}$ and when $X_{\beta}=0, X_{2 \beta}^{\prime}$ $=\operatorname{Ad}(a m) X_{2 \beta}$. Now $w^{*-1} u^{\prime} w^{*}=\exp \left(\sum_{\alpha \in R^{+}} Y_{w(\alpha)}^{\prime}\right) \in P_{0}$, where $Y_{w(\alpha)}^{\prime}=\left(\operatorname{Ad} w^{*}\right) X_{\alpha}^{\prime}$ belongs to the $w(\alpha)$-root space of $A$. Hence $w(\alpha) \in R^{+}$unless $X_{\alpha}^{\prime}=0$. But $X_{\beta}^{\prime}+X_{2 \beta}^{\prime} \neq 0$ for every simple $\beta$; therefore $w$ stabilizes the positive Weyl chamber. Since the Weyl group acts simply transitively on the Weyl chambers of $A, w$ is an identity element of $\mathrm{W}(G, A)$. Therefore $w^{*} \in \mathrm{Z}_{\mathrm{G}}(A) \subset P_{0}$. Hence $g N g^{-1}=N$. Since any two maximal unipotent subgroups of $G$ are conjugate, (c) $\Rightarrow$ (a).

(4.4) Remark. Suppose $G$ is a semisimple group of $\mathbb{R}$-rank 1 . Then in the notation of the Theorem $4.3, R^{+}$contains only one simple root. Therefore, any nontrivial unipotent element of $G$ is regular.

(4.5) Lemma. Let $G$ be a semisimple group with trivial center and let $H \subset G$ be a reductive algebraic stibgroup of $G$. If $H$ contains a regular unipotent element of $G$ then $\mathrm{Z}_{\mathrm{G}}(H)$ is compact.

Proof. Let the notation be as in Theorem 4.3. Let $u \in H$ be a regular unipotent element of $G$. We may assume that $u \in N$. Then $Z_{G}(u) \subset P_{0}$. By Theorem 4.3(c), it is easy to see that $Z_{G}(u) \subset M N$. Therefore $Z_{G}(H) \subset M N$. But $Z_{G}(H)$ is reductive group (cf. $[18$, Sect. P.2.6]). Hence it must be compact.

\section{Union of lower dimensional homogeneous closures}

Let $G$ be a semisimple Lie group with trivial center and no compact factors, $\Gamma$ be a lattice in $G$ and $U=\left\{u_{t}\right\}_{\in \mathbb{R}}$ be a regular unipotent one-parameter subgroup of $G$.

Let $X=G / \Gamma$ and $Y=\{y \in X \mid F y$ is closed for a connected Lie subgroup $F$ of $G$ such that $\operatorname{dim} F<\operatorname{dim} G$ and $U C F\}$.

Take $y \in Y$ and let $F$ be the smallest Lie subgroup of $G$ such that $U \subset F$ and $F y$ is closed. Then $\operatorname{dim} F<\operatorname{dim} G$. By Theorem 2.3 there exists $x \in F y$ such that $\overline{U x}=F x$ $=F y$. By Proposition 3.10, either 1) $F$ is a connected reductive group with compact center or 2) there exists a proper parabolic subgroup $P$ of $G$ such that (a) $U C^{0} P$, (b) ${ }^{0} P x$ is closed and (c) $R_{u}(P) x$ is compact.

Consider the first possibility. Let $F^{\text {nc }}$ denote the maximal connected normal semisimple subgroup of $F$ with no compact factors. Let $H=\mathrm{N}_{\mathrm{G}}\left(F^{\mathrm{nc}}\right)$. Then $H$ is an open subgroup of $\mathbb{R}$-points of a real algebraic group. Hence $H$ has finitely many components (cf. [18, Prelim. 2.4]). Since $F^{\text {nc }}$ is connected and semisimple, $H^{0}$ $C \mathrm{Z}_{\mathrm{G}}\left(F^{\mathrm{nc}}\right) F^{\mathrm{nc}}$. By Lemma $4.5, \mathrm{Z}_{\mathrm{G}}\left(F^{\mathrm{nc}}\right)$ is compact. Therefore $H x$ is closed and $H^{\mathrm{nc}}=F^{\mathrm{nc}}$.

Let $\mathscr{R}$ be the set of all pairs of the form $(H, x)$, where $x \in Y$ and $H$ is a reductive subgroup of $G$ such that (a) $U \subset H$, (b) $H x$ is closed, (c) $\operatorname{dim} H<\operatorname{dim} G$ and (d) there exists a connected reductive subgroup $F$ with compact center such that $H=\mathrm{N}_{\mathrm{G}}\left(F^{\mathrm{nc}}\right)$ and $\overline{U x}=F x$.

Let $\mathscr{P}$ be the set of all pairs of the form $\left({ }^{0} P, x\right)$, where $x \in Y$ and $P$ is a parabolic subgroup of $G$ such that (a) $U \subset H$, (b) $H x$ is closed, (c) $\operatorname{dim} H<\operatorname{dim} G$, and (d) there

(5.1) Remark. Let $\mathscr{F}=\mathscr{P} \cup \mathscr{R}$. Then from the above discussion,

$$
Y=\bigcup_{(F, x) \in \mathscr{F}} F x \text {. }
$$


(5.2) Lemma. There is a countable subset $\mathscr{F}^{*}$ of $\mathscr{F}$ such that if $\left(F^{\prime}, x^{\prime}\right) \in \mathscr{F}$ then there exist $\mathrm{g} \in G$ and $(F, x) \in \mathscr{F}^{*}$ such that $F^{\prime}=\mathrm{gFg}^{-1}$ and $x^{\prime}=\mathrm{gx}$.

Proof. Let $\mathscr{D}$ be the set of connected Lie subgroups of $G$ such that if $A \in \mathscr{D}$ then (i) either $A$ is a reductive group with compact center or $A$ is unipotent subgroup of $G$ and (ii) $A \cap \Gamma$ is a Zariski dense lattice in $A$. In both cases $A$ is the identity component of a real algebraic group. Moreover $A \cap \Gamma$ is finitely generated (cf. [18, Sects. $2.10,13.20,13.25]$ ). Thus $A$ is completely determined by finitely many elements of $\Gamma$. Hence $\mathscr{D}$ is countable.

Let $\left(H_{1}, x_{1}\right),\left(H_{2}, x_{2}\right) \in \mathscr{R}$. Let $F_{1}$ and $F_{2}$ be closed connected subgroups such that $\overline{U x_{1}}=F_{1} x_{1}$ and $\overline{U x_{2}}=F_{2} x_{2}$. Let $g_{1}, g_{2} \in G$ be such that $x_{1}=g_{1} \Gamma$ and $x_{2}=g_{2} \Gamma$. Then by the definition of $\mathscr{R}$ and Corollary $2.13, g_{1} F_{1} g_{1}^{-1}, g_{2} F_{2} g_{2}^{-1} \in \mathscr{D}$. Suppose if $g_{1} F_{1} g_{1}^{-1}=g_{2} F_{2} g_{2}^{-1}$ then $g_{1} \mathrm{~N}_{\mathrm{G}}\left(F_{1}^{\mathrm{nc}}\right) g_{1}^{-1}=g_{2} \mathrm{~N}_{\mathrm{G}}\left(F_{2}^{\mathrm{nc}}\right) g_{2}^{-1}$. By definition $H_{1}$ $=\mathrm{N}_{\mathrm{G}}\left(F_{1}^{\mathrm{nc}}\right)$ and $H_{2}=\mathrm{N}_{\mathrm{G}}\left(F_{2}^{\mathrm{nc}}\right)$. If we put $g=g_{1} g_{2}^{-1}$ then $H_{1}=g H_{2} g^{-1}$ and $x_{1}=g x_{2}$. Thus by countability of $\mathscr{D}$ we can choose a countable subset $\mathscr{R}^{*}$ of $\mathscr{R}$ with the property that for every $\left(F^{\prime}, x^{\prime}\right) \in \mathscr{R}$, there exits $g \in G$ such that $\left(g^{-1} F^{\prime} g, g^{-1} x^{\prime}\right) \in \mathscr{R}^{*}$.

Take $\left({ }^{0} P, x\right) \in \mathscr{P}$. Then $\mathrm{R}_{\mathrm{u}}(P) x$ is compact. Take $g \in G$ such that $x=g \Gamma$. Then by Theorem 2.1 in $[18], g R_{u}(P) g^{-1} \in \mathscr{D}$. Since $P$ is a parabolic subgroup, $P=\mathbf{N}_{\mathrm{G}}\left(\mathbf{R}_{\mathrm{u}}(P)\right)$. Now argue as in the above case.

(5.3) Notation. For a Lie subgroup $F$ of $G$ containing $U$ define

$$
L(F)=\left\{g \in G \mid g F g^{-1} \supset U\right\} .
$$

Note that $\mathrm{N}_{\mathrm{G}}(U) L(F) \mathrm{N}_{\mathrm{G}}(F)=L(F)$.

(5.4) Remark. Let $\left({ }^{0} P, x\right) \in \mathscr{P}$. If $g \in L\left({ }^{0} P\right)$ then $U \subset P \cap g P g^{-1}$. Since $U$ contains a regular unipotent element of $G$, by Theorem $4.3, L\left({ }^{\circ} P\right)=P$.

(5.5) Notations. Define $\mathscr{F}_{0}=\mathscr{P} \cap \mathscr{F} *$ and for all $n \in \mathbb{N}$ define

$$
\mathscr{F}_{n}=\left\{(H, x) \in \mathscr{R} \cap \mathscr{F}^{*} \mid \operatorname{dim}\left(H^{\mathrm{nc}}\right)=n\right\} \text {. }
$$

Put $Y_{-1}=\emptyset$ and for all $n \in \mathbb{N} \cup\{0\}$ define

$$
Y_{n}=Y_{n-1} \bigcup\left(\bigcup_{(F, x) \in F_{n}} L(F) x\right)
$$

The next corollary is a direct consequence of the above discussion.

(5.6) Corollary. $Y_{n}=Y$ for all $n \geqq \operatorname{dim} G-1$.

\section{Proof of the main theorem}

We follow the notations of Sect. 5. To prove the main theorem (for $G$ as above) it is enough to prove the following.

(6.1) Theorem. Let $n \in \mathbb{N} \cup\{0,-1\}$. Given $\varepsilon>0$ and a point $y \in X \backslash Y_{n}$ there exists $a$ compact subset $K \subset X \backslash Y_{n}$ such that for all $T>0$,

$$
(1 / T) \ell\left\{t \in[0, T] \mid u_{t} y \in K\right\}>1-\varepsilon \text {. }
$$

For $n=-1$, that is when $Y_{n}=\emptyset$, the theorem is essentially proved in $[5,6]$ and the above form is deduced in Proposition 1.8 of [8]. For $n=0$ it is proved in the Appendix of [8]. In this paper we exploit the techniques of [8] to study the case of $n \in \mathbb{N}$. We shall assume the theorem for $n=-1$ and give a proof by induction on $n \in \mathbb{N} \cup\{0\}$. 
(6.2) Lemma. For each $(F, x) \in \mathscr{F}$ there is a finite dimensional real vector space $E$ equipped with a linear $G$ action and a point $p \in E$ such that

(a) $F$ is the isotopy subgroup of $p$,

(b) if $(F, x) \in \mathscr{R}$ then $G p$ is closed, and

(c) if $(F, x)=\left({ }^{0} P, x\right) \in \mathscr{P}$ then $a p=\operatorname{det}\left(\left.\operatorname{Ad} a\right|_{w}\right) p$ for all $a \in P$, where $\mathrm{w}$ is the Lie subalgebra corresponding to $\mathrm{R}_{\mathrm{u}}(P)$.

Proof. First note that $G=\mathbf{G}_{\mathbf{R}}^{0}$ for an algebraic $\mathbb{R}$-group $\mathbf{G}$. Suppose $(F, x) \in \mathscr{R}$. By the definition $F=G \cap \mathbf{H}$ for a reductive algebraic $\mathbb{R}$-subgroup $\mathbf{H} \subset \mathbf{G}$. Now Proposition 7.7 in [1] provides such a representation.

For $(F, x)=\left({ }^{0} P, x\right) \in \mathscr{P}$ such a representation is constructed explicitly in the proof of Lemma 3.4.

(6.3) Lemma. Let $\left({ }^{\circ} P, x\right) \in \mathscr{P}$ and $(E, p)$ be a linear G-space with a distinguished vector as in Lemma 6.2. Let $W=\mathrm{R}_{\mathrm{u}}(P)$ and $\mathrm{w}$ be the Lie subalgebra corresponding to $W$. Then the following holds.

1. If $a \in P$ then $\operatorname{Vol}\left(W / W_{a x}\right)=\operatorname{det}\left(\left.\operatorname{Ad} a\right|_{w}\right) \mid \operatorname{Vol}\left(W / W_{x}\right)$, the volumes of the quotients being understood to be relative to a fixed Haar measure on $W$.

2. If $a x=a^{\prime} x$ for some $a, a^{\prime} \in P$ then $a p= \pm a^{\prime} p$.

3. For a sequence $\left\{a_{i}\right\}_{i \in \mathbf{N}} \subset P$, if $a_{i} p \rightarrow 0$ as $i \rightarrow \infty$ then no subsequence of $\left\{a_{i} x\right\}_{i \in \mathbf{N}}$ converges in $X$.

Proof. Let $\sigma$ be a Haar measure on $W$. Let $\mathscr{S}$ be a fundamental domain for the lattice $W_{x}$ in $W$. Then $\operatorname{Vol}\left(W / W_{x}\right)=\sigma(\mathscr{S})$. Since $W_{a x}=a W_{x} a^{-1}$, the set $a \mathscr{S} a^{-1}$ is a fundamental domain for $W_{a x}$. Therefore $\operatorname{Vol}\left(W / W_{a x}\right)=\sigma\left(a \mathscr{S} a^{-1}\right)$ $=\left|\operatorname{det}\left(\left.\operatorname{Ad} a\right|_{w}\right)\right| \sigma(\mathscr{S})$. Hence 1) holds.

Now 2) is an immediate consequence of 1) and the condition (c) of Lemma 6.2.

Now suppose that $a_{i} p \rightarrow 0$. Then by 1$), \operatorname{Vol}\left(W / W_{a_{i} x}\right) \rightarrow 0$. Since $W_{a_{i x}}=a_{i} W_{x} a_{i}^{-1}$, there exists a sequence $\left\{\gamma_{i}\right\}_{i \in \mathrm{N}} \subset W_{x} \subset G_{x}$, such that $\gamma_{i} \neq e$ and $a_{i} \gamma_{i} a_{i}^{-1} \rightarrow e$. Hence $\left\{a_{i} x\right\}_{i \in \mathrm{N}}$ is divergent in $X$ (cf. [18, Sect. 1.12]). This proves 3).

(6.4) Remark. In the notation of Lemma 6.3, $0 \in \overline{P p}$. Therefore if $X$ is compact then $\mathscr{P}=\emptyset$.

The following lemma will enable us to apply induction in the proof of Theorem 6.1.

Lemma. Let $(H, x) \in \mathscr{R}$. Define

$$
Z=\left\{z \in Y \mid z \in l_{1} H x \cap l_{2} H x \text { and } l_{1} H \neq l_{2} H \text { for some } l_{1}, l_{2} \in L(H)\right\} .
$$

Then $Z \subset Y_{n-1}$, where $n=\operatorname{dim}\left(H^{\mathrm{nc}}\right)$.

Proof. Let $z \in l_{1} H x \cap l_{2} H x$ be such that $l_{1} H \neq l_{2} H$, where $l_{1}, l_{2} \in L(H)$. Now $l_{i} H x$ $=\left(l_{i} H l_{i}^{-1}\right)\left(l_{i} x\right)$ for $i=1,2$. Since $H x$ is closed, by Lemma 2.2 if $F=\left(l_{1} H l_{1}^{-1}\right)$ $\cap\left(l_{2} H l_{2}^{-1}\right)$ then $F z$ is closed. By the definition of $L(H), U \subset F$. Let $F_{0}$ be the smallest Lie subgroup of $G$ such that $U \subset F_{0}$ and $F_{0} z$ is closed. If $F_{0}$ is not reductive then by definition $z \in Y_{0}$.

Suppose $F_{0}$ is reductive. Since $F_{0} \subset F$, we have $F_{0}^{\mathrm{nc}} \subset\left(l_{1} H^{\mathrm{nc}} l_{1}^{-1}\right)$. If $\operatorname{dim}\left(F_{0}^{\mathrm{nc}}\right)$ $<\operatorname{dim}\left(H^{\text {nc }}\right)$ then $z \in Y_{n-1}$.

Now suppose $\operatorname{dim}\left(F_{0}^{\mathrm{nc}}\right)=\operatorname{dim}\left(H^{\mathrm{nc}}\right)$. Since $F_{0}^{\mathrm{nc}}$ and $H^{\mathrm{nc}}$ are connected, $F_{0}^{\mathrm{nc}}=l_{1} H^{\mathrm{nc}} l_{1}^{-1}=l_{2} H^{\mathrm{nc}} l_{2}^{-1}$. Therefore, $l_{1}^{-1} l_{2} \in \mathrm{N}_{\mathrm{G}}\left(H^{\mathrm{nc}}\right)=H$, a contradiction. This completes the proof.

The following notations are used in Sect. 6.8 to Sect. 6.13.

(6.6) Notations. Let $(F, x) \in \mathscr{F}$ and $(E, p)$ be a linear $G$-space with a distinguished vector, satisfying the properties mentioned in Lemma 6.2. 
Let

$$
L=\{v \in E \mid U v=v\}
$$

be the fixed point space of $U$ in $E$. For a subset $S C E$ define

$$
S(x)=\{g x \in X \mid g \in G \text { and } g p \in S\} .
$$

(6.7) Remark. If $g \in G$ and $g p \in L$ then $U(g p)=g p$. Therefore $g^{-1} U g \subset F$ and hence $g \in L(F)$. Thus $L(x)=L(F) x$.

(6.8) Lemma. Let $\Delta=\{(g x, g F) \in X \times G / F \mid g \in G\}$. Let $\psi: \Delta \rightarrow X \times E$ be the map defined by $\psi(g x, g F)=(g x, g p)$ for all $g \in G$. Then $\psi$ is injective and proper.

Proof. First note that $\psi$ is well defined and injective. Also $\psi$ is $G$-equivariant with respect to the obvious $G$-actions.

Suppose $(F, x) \in \mathscr{R}$. In this case $G p$ is closed. Therefore the map $\alpha: G / F \rightarrow E$ defined by $\alpha(g F)=g p$ for all $g \in G$ is proper. Hence $\psi$ is proper.

Suppose $(F, x)=\left({ }^{0} P, x\right) \in \mathscr{P}$. By Iwasawa decomposition there is a maximal compact subgroup $K_{0}$ of $G$ such that $G=K_{0} P$. Let $\Delta_{P}=\left\{\left(a x, a^{0} P\right) \in \Delta \mid a \in P\right\}$. Then $\Delta=\mathrm{K}_{0} \Delta_{P}$. Let $\psi_{\mathrm{r}}: \Delta_{P} \rightarrow X \times E$ be the restriction of $\psi$ to $\Delta_{p}$. Since $K_{0}$ is compact and $\psi$ is $G$-equivariant, it is enough to show that $\psi_{\mathrm{r}}$ is proper. Since $\overline{\boldsymbol{P p}}$ $=P p \cup\{0\}$, the properness of $\psi_{\mathrm{r}}$ follows immediately from 3) of Lemma 6.3.

(6.9) Corollary. For $y \in X$, the set $S=\{g p \in E \mid g \in G$ and $y=g x\}$ is closed.

Proof. Since $F x$ is closed, the set $G_{x} F$ is closed in $G$. If $y=g x$ for some $g \in G$ then the set $\Delta_{y}=\left(y, g G_{x} F\right) \subset \Delta$ is closed. For the map $\psi$ as in Lemma $6.8, \psi\left(\Delta_{y}\right)=(y, S)$. Since $\psi$ is proper, $S$ is closed.

(6.10) Lemma. Let $\varphi: G / F_{x} \rightarrow X \times E$ be the map defined by $\varphi\left(g F_{x}\right)=(g x, g p)$ for all $g \in G$. Then $\varphi$ is proper.

Proof. Let $\pi: G / F_{x} \rightarrow X \times G / F$ be the map defined by $\pi\left(g F_{x}\right)=(g x, g F)$ for all $g \in \dot{G}$. Then $\varphi=\psi \circ \pi$. In view of Lemma 6.8, it is enough to prove that $\pi$ is proper.

Let $\left\{g_{i}\right\} \subset G$ be a sequence such that $\left\{g_{i} x\right\}$ and $\left\{g_{i} F\right\}$ are convergent. Then there are sequences $\left\{\delta_{i}\right\} \subset G_{x}$ and $\left\{f_{i}\right\} \subset F$ such that $\left\{g_{i} \delta_{i}\right\}$ and $\left\{g_{i} f_{i}\right\}$ converge in $G$. Therefore $\left\{f_{i}^{-1} \delta_{i}\right\}=\left\{\left(g_{i} f_{i}\right)^{-1}\left(g_{i} \delta_{i}\right)\right\}$ converges in $G$. Hence $f_{i}^{-1} x$ converges in $X$. Since $F x$ is closed, the map i: $F / F_{x} \rightarrow X$ given by $\mathbf{i}\left(f F_{x}\right)=f x$ for all $f \in F$, is a homeomorphism on to its image. Therefore $\left\{f_{i}^{-1} F_{x}\right\}$ converges in $F / F_{x}$ and hence $\left\{g_{i} F_{x}\right\}=\left\{\left(g_{i} f_{i}\right) f_{i}^{-1} F_{x}\right\}$ converges in $G / F_{x}$. This shows that $\pi$ is proper.

(6.11) Proposition. Let $n \in \mathbb{N} \cup\{0\}$ and $(F, x) \in \mathscr{F}_{n}$. Let $K$ be a compact subset of $X \backslash Y_{n-1}$ and $C$ be a compact subset of $L$. Then there exists a neighbourhood $\Omega$ of $C$ in $E$ such that if $g, g^{\prime} \in G, g x=g^{\prime} x \in K$ and $g p, g^{\prime} p \in \Omega$ then $g p= \pm g^{\prime} p$.

Proof. Suppose this is not true. Then there exist sequences $\left\{g_{i}\right\}$ and $\left\{g_{i}^{\prime}\right\}$ contained in $G$ and points $y \in K$ and $c, c^{\prime} \in C$ such that the following holds. (i) For each $i \in \mathbb{N}, y_{i}=g_{i} x=g_{i}^{\prime} x \in K$ and $g_{i} p \pm g_{i}^{\prime} p$. (ii) As $i \rightarrow \infty, y_{i} \rightarrow y, g_{i} p \rightarrow c$ and $g_{i}^{\prime} p \rightarrow c^{\prime}$.

Since the map $\varphi$ as in Lemma 6.10 is proper, the sequences $\left\{g_{i} F_{x}\right\}$ and $\left\{g_{i}^{\prime} F_{x}\right\}$ have convergent subsequences in $G / F_{x}$. Passing to subsequences and replacing $g_{i}$ and $g_{i}^{\prime}$ by appropriate elements of $g_{i} F_{x}$, and $g_{i}^{\prime} F_{x}$ we may assume that $g_{i} \rightarrow g$ and $g_{i}^{\prime} \rightarrow g^{\prime}$ for some $g, g^{\prime} \in G$. Now $g_{i}^{-1} g_{i}^{\prime} \in G_{x}$ for all $i \in \mathbb{N}$. Since $g_{i}^{-1} g_{i}^{\prime} \rightarrow g^{-1} g^{\prime}$ and $G_{x}$ is discrete, there exists $\delta \in G_{x}$ such that $g_{i}^{\prime}=g_{i} \delta$ for all large $i$. Since $g_{i} p \neq \pm g_{i}^{\prime} p$ $= \pm g_{i} \delta p$, we have $\delta p \neq \pm p$. Hence $g p \neq \pm g^{\prime} p$. Since $g p=c, g^{\prime} p=c^{\prime} \in L$, by Remark 6.7, $g, g^{\prime} \in L(F)$. Also $y=g x=g^{\prime} x$.

Suppose $(F, x)=\left({ }^{0} P, x\right) \in \mathscr{P}$. By Remark $\left.5.4, g, g^{\prime} \in L^{0} P\right)=P$. Now $g x=g^{\prime} x$ but $g p \neq \pm g^{\prime} p$. This contradicts 2) of Lemma 6.3. 
Suppose $(F, x)=(H, x) \in \mathscr{R}$. Since $g, g^{\prime} \in L(H), y \in g H x \cap g^{\prime} H x$ and $g H \neq g^{\prime} H$, by Lemma $6.5, y \in Y_{n-1}$. This contradicts the fact that $y \in K$ and $K \cap Y_{n-1}=\emptyset$. This completes the proof.

The following Lemma about polynomial growth is very useful in studying the dynamical behaviour of orbits of a unipotent flow in a vector space.

(6.12) Lemma [8, Lemma A.8]. Let $n \in \mathbb{N}$ and $\varepsilon>0$ be given. Then for any $\alpha>0$ there exists $a \beta \in(0, \alpha)$ and for any $\beta>0$ there exists an $\alpha>\beta$ such that the following condition is satisfied if $\varphi$ is a polynomial on $\mathbb{R}$ of degree atmost $n$ such that $|\varphi(0)| \geqq \alpha$ then there exists $t \in(1,1+\varepsilon)$ such that $|\varphi(t)| \geqq \beta$.

(6.13) Proposition. Let $n \in \mathbb{N} \cup\{0\}$ and $(F, x) \in \mathscr{F}_{n}$. Let $y \in X \backslash Y_{n}, K$ be a compact subset of $X \backslash Y_{n-1}$ and $C$ be a compact subset of $L$. Then, given $\varepsilon>0$, there exists $a$ neighbourhood $\Psi$ of $C$ in $E$ such that for all $T>0$,

$$
\ell\left\{t \in[0, T] \mid u_{t} y \in \Psi(x) \cap K\right\}<\varepsilon T .
$$

Proof. Let $L^{\perp}$ be a complementary subspace of $L$ in $E$. Let $c=\operatorname{dim} L, d=\operatorname{dim} L^{\perp}$ and $m=c+d=\operatorname{dim} E$. Let $\left\{e_{1}, \ldots, e_{c}\right\}$ be a basis of $L$ and $\left\{f_{1}, \ldots, f_{d}\right\}$ be a basis of $L^{\perp}$. For $r>0$ define

$$
I(r)=\left\{\sum_{i=1}^{c} \xi_{i} e_{i}|| \xi_{i} \mid<r \text { for all } 1 \leqq i \leqq c\right\} \subset L
$$

and

$$
J(r)=\left\{\sum_{j=1}^{d} \xi_{j} f_{j}|| \xi_{j} \mid<r \text { for all } 1 \leqq j \leqq d\right\} \subset L^{\perp}
$$

Let $\beta>0$ be such that $I(\beta) \supset C$. By Lemma 6.12 there exists $\alpha>\beta$ such that if $\varphi$ is a polynomial on $\mathbb{R}$ of degree atmost $m$ and if $|\varphi(0)| \geqq \alpha$ then there exists $t \in\left(1,1+\varepsilon /\left(2 m^{2}\right)\right)$ such that $|\varphi(t)| \geqq \beta$.

Let $C^{\prime}=\overline{I(\alpha)}$. By Proposition 6.11 there exists a neighbourhood $\Omega^{\prime}$ of $C^{\prime}$ in $E$ such that if $g, g^{\prime} \in G, g x=g^{\prime} x \in K$ and $g p, g^{\prime} p \in \Omega^{\prime}$, then $g p= \pm g^{\prime} p$. Since $y \notin Y_{n} \supset L(x)$, by Corollary 6.9 there exists $a>0$ such that if $\Omega=I(\alpha) \times J(a)$ then $y \notin \Omega(x)$ and $\Omega \subset \Omega$.

By Lemma 6.12 there exists $0<b<a$ such that if $\varphi$ is a polynomial on $\mathbb{R}$ of degree atmost $m$ and if $|\varphi(0)| \geqq a$ then there exists $t \in\left(1,1+\varepsilon /\left(2 m^{2}\right)\right)$ such that $|\varphi(t)| \geqq b$. Let $\Psi=I(\beta) \times J(b)$. Then $\Psi \subset \Omega$ and $\Psi$ is a neighbourhood of $C$ in $E$.

For $g \in G$ and $T>0$ define,

$$
A(g)=\left\{t \in(0, T) \mid u_{i}(g p) \in \Omega\right\} .
$$

Note that $A(g)$ is a union of open intervals.

Step 1. If $\mathrm{gp} \notin \Omega, I=(r, s)$ is a connected component of $A(g)$ and $s^{\prime} \in(r, s]$ then

$$
\ell\left\{t \in\left[r, s^{\prime}\right] \mid u_{t}(g x) \in \Psi(x) \cap K\right\}<\varepsilon\left(s^{\prime}-r\right) .
$$

Proof. Since $g p \notin \Omega, u_{r}(g p) \in \Omega \backslash \Omega$. Therefore replacing $g$ by $u_{r} g$, we may assume that $r=0$. Now if $t \in[0, s]$ and $u_{t}(g x) \in \Psi(x) \cap K$ then $u_{t}(g p) \in \Omega$ and there exists $g^{\prime} \in G$ such that $g^{\prime} x=\left(u_{t} g\right) x$ and $g^{\prime} p \in \Psi$. By the choice of $\Omega,\left(u_{t} g\right) p= \pm g^{\prime} p$. Since $\Psi$ is symmetric about the origin, $u_{t}(g p) \in \Psi$. Therefore

$$
\ell\left\{t \in\left[0, s^{\prime}\right] \mid u_{t}(g x) \in \Psi(x) \cap K\right\}<\ell\left\{t \in\left[0, s^{\prime}\right] \mid u_{t}(g p) \in \Psi\right\} .
$$

Note that $U$ acts by unipotent linear transformations on $E$ (cf. [21, Sect. 3.4.2]). Therefore there exist polynomials $\varphi_{1}, \ldots, \varphi_{c}$ and $\psi_{1}, \ldots, \psi_{d}$ on $\mathbb{R}$ of degree atmost 
$m(=\operatorname{dim} E)$ such that for all $t \in \mathbb{R}$,

$$
u_{t}(g p)=\sum_{i=1}^{c} \varphi_{i}(t) e_{i}+\sum_{j=1}^{d} \psi_{j}(t) f_{j} .
$$

Let $A_{1}=\left\{t \in\left(0, s^{\prime}\right) \mid u_{t}(g p) \in \Psi\right\}$. Then $A_{1}$ is open. Let $\left(a_{0}, b_{0}\right)$ be a connected component of $A_{1}$. Since $g p \notin \Psi, u_{a_{0}}(g p) \in \vec{\Psi} \backslash \Psi$. Therefore there exist $i \in\{1, \ldots, c\}$ such that $\left|\varphi_{i}\left(a_{0}\right)\right|=\beta$ or $j \in\{1, \ldots, d\}$ such that $\left|\psi_{j}\left(a_{0}\right)\right|=b$. Hence $A_{1}$ consists of atmost $2 \mathrm{~m}^{2}$ connected components. Now $A_{1}=\left(a_{1}, b_{1}\right) \cup \ldots \cup\left(a_{l}, b_{l}\right)$, where $l \leqq 2 m^{2}$ and $b_{k} \leqq a_{k+1}$ for $1 \leqq k<l$.

Take $k \in\{1, \ldots, l\}$. Since $u_{r}(g p) \notin \Omega$, there exist $i \in\{1, \ldots, c\}$ such that $\left|\varphi_{i}(0)\right| \geqq \alpha$ or $j \in\{1, \ldots, d\}$ such that $\left|\psi_{j}(0)\right| \geqq a$. Now by the choice of $\alpha$ and $b$, there exists $t \in\left(1,1+\varepsilon /\left(2 m^{2}\right)\right)$ such that $\varphi_{i}\left(t a_{k}\right) \geqq \beta$ or $\psi_{j}\left(t a_{k}\right) \geqq b$. Therefore, $0<b_{k}<t a_{k}$. Hence $\left(b_{k}-a_{k}\right)<\left(\varepsilon /\left(2 m^{2}\right)\right) a_{k}<\left(\varepsilon /\left(2 m^{2}\right)\right) s^{\prime}$. Thus

$$
\ell\left\{t \in\left[0, s^{\prime}\right] \mid u_{t}(g p) \in \Psi\right\}=\ell\left(A_{1}\right)=\sum_{k=1}^{l}\left(b_{k}-a_{k}\right)<\varepsilon s^{\prime} .
$$

Since $r=0$, Step 1 follows from (1) and (2).

Let

$$
G_{x, y}=\{g \in G \mid g x=y\} .
$$

Fix $T>0$. Let $\mathscr{C}$ be the collection of pairs of the form $(I, g)$, where $g \in G_{x, y}$ and $I$ is a connected component of $A(g)$. For an interval $I=(r, s) \subset \mathbb{R}$ define $J(I)=\left(r, s^{\prime}\right]$, where

$$
s^{\prime}=\sup \left\{t \in[r, s] \mid u_{t} y \in K\right\} .
$$

Step 2. We can choose a subcollection $\mathscr{C}_{0} \subset \mathscr{C}$ satisfying the following conditions.

1. $\left\{t \in[0, T] \mid u_{t} y \in \Psi(x) \cap K\right\} \subset \bigcup \bigcup J(I)$.

2. For $\left(I_{1}, g_{2}\right),\left(I_{2}, g_{2}\right) \in \mathscr{C}_{0}$ if $\left(I_{1}, g_{1}\right) \neq\left(I_{2}, g_{2}\right)$ then $J\left(I_{1}\right) \cap J\left(I_{2}\right)=\emptyset$.

Proof. For $t>0$ if $u_{t} y \in K \cap \Psi(x)$ then there exists $(I, g) \in \mathscr{C}$ such that $t \in J(I)$. Suppose $J\left(I_{1}\right) \cap J\left(I_{2}\right) \neq \emptyset$ for some $\left(I_{1}, g_{1}\right),\left(I_{2}, g_{2}\right) \in \mathscr{C}$. Then there exists $t_{0} \in \bar{I}_{1} \cap \overline{I_{2}}$ such that $u_{t_{0}} y \in K$ and $u_{t_{0}} g_{1} p, u_{t_{0}} g_{2} p \in \bar{\Omega} \subset \Omega^{\prime}$. By the choice of $\Omega^{\prime}$, we have $u_{t_{0}}\left(g_{1} p\right)= \pm u_{t_{0}}\left(g_{2} p\right)$. Thus $u_{t}\left(g_{1}\right) p= \pm u_{t}\left(g_{2}\right) p$ for all $t \in \mathbb{R}$. Since $\Omega$ is symmetric about the origin, $A\left(g_{1}\right)$ $=A\left(g_{2}\right)$ and hence $I_{1}=I_{2}$. Now it is clear how to choose $\mathscr{C}_{0}(\mathscr{C}$ so that 1) and 2) are satisfied.

Thus by Step 1 and Step 2,

$$
\ell\left\{t \in[0, T] \mid u_{t} y \in \Psi(x) \cap K\right\} \leqq \varepsilon\left(\sum_{(I, g) \in \Psi_{0}} \ell(J(I))\right) \leqq \varepsilon T .
$$

Proof of Theorem 6.1. For $n=-1$ the theorem is same as Proposition 1.8 in [8].

Take $n \in \mathbb{N} \cup\{0\}$. Then by Notations 5.5 and Remark 6.7,

$$
Y_{n}=Y_{n-1} \cup\left(\bigcup_{(F, x) \in \mathscr{F}_{n}} L(x)\right) \text {. }
$$

Now $\mathscr{F}_{n}$ is countable and for each $(F, x) \in \mathscr{F}_{n}$ the set $L$ is a countable union of compact subsets. Therefore, for each $i \in \mathbb{N}$ we can choose a 3-tuple $\left(F_{i}, x_{i}, C_{i}\right)$ such that (a) $\left(F_{i}, x_{i}\right) \in \mathscr{F}_{n}$, (b) $C_{i}$ is a compact subset of $L_{i}$, and (c) $\bigcup_{(F, x) \in F_{n}} L(x)$ $=\bigcup_{i \in \mathbb{N}} C_{i}\left(x_{i}\right)$.

By induction there exists a compact subset $K^{\prime} \subset X \backslash Y_{n-1}$ such that for all $T>0$,

$$
\ell\left\{t \in[0, T] \mid u_{t} y \in K^{\prime}\right\}>(1-\varepsilon / 2) T .
$$


By Proposition 6.13, for each $i \in \mathbb{N}$ there exists a neighbourhood $\Psi_{i}$ of $C_{i}$ in $E_{i}$ such that for all $T>0$,

$$
\ell\left\{t \in[0, T] \mid u_{t} y \in \Psi_{i}(x) \cap K^{\prime}\right\}<\left(\varepsilon / 2^{i+1}\right) T .
$$

Let $K=K^{\prime} \backslash\left(\bigcup_{i \in \mathbb{N}} \Psi_{i}(x)\right)$. Then $K \subset X \backslash Y_{n}$. By (3) and (4),

$$
(1 / T) \ell\left\{t \in[0, T] \mid u_{t} y \in K\right\}>1-\varepsilon / 2-\sum_{i \in \mathbb{N}} \varepsilon / 2^{i+1}=1-\varepsilon .
$$

(6.14) Reduction to semisimple case. Let $G$ be a connected reductive Lie group; that is, the adjoint action of $G$ on its lie algebra is completely reducible. Let $G^{*}$ be the adjoint group of $G$. Then $G^{*}$ admits a direct product decomposition $G^{*}=C \cdot G^{\prime}$, where $C$ and $G^{\prime}$ are normal (and hence semisimple) subgroups of $G^{*}, C$ is compact and $G^{\prime}$ is a semisimple group with trivial center and no compact factors. Let $\varrho: G \rightarrow G^{\prime}$ be the projection homomorphism of $G$ onto $G^{\prime}$.

Let $\Gamma$ be a lattice in $G$. Let $\bar{\varrho}: X=G / \Gamma \rightarrow X^{\prime}=G^{\prime} / \varrho(\Gamma)$ be the map defined by $\bar{\varrho}(\Gamma)$ $=\varrho(g) \varrho(\Gamma)$ for all $g \in G$.

(6.15) Lemma [2, Lemma 9.1]. $\varrho(\Gamma)$ is a lattice in $G^{\prime}$ and $\varrho$ is proper.

Let $U$ be a regular unipotent one parameter subgroup of $G$. Then $U^{\prime}=\varrho(U)$ is a regular unipotent one parameter subgroup of $G^{\prime}$. Let $Y=\{y \in X \mid F y$ is closed for a connected Lie subgroup $F$ of $G$ such that $\operatorname{dim} F<\operatorname{dim} G$ and $U \subset F\}$. Let $Y^{\prime} \subset X^{\prime}$ be similarly defined with respect to $U^{\prime} \subset G^{\prime}$.

(6.16) Lemma. (i) $\bar{\varrho}^{-1}\left(Y^{\prime}\right) \subset Y$. (ii) If $Y \neq X$ then $\bar{\varrho}(Y)=Y^{\prime}$.

Proof. (i) follows from properness of $\bar{\varrho}$.

Now let $y \in Y$. Let $F$ be a connected Lie subgroup of $G$ such that $U C F, \operatorname{dim} F$ $<\operatorname{dim} G$ and $F y$ is closed. Since $\bar{\varrho}$ is a proper map, if $F^{\prime}=\varrho(F)$ and $y^{\prime}=\bar{\varrho}(y)$ then $F^{\prime} y^{\prime}$ $=\bar{\varrho}(F y)$ is closed. Also $U^{\prime} C F^{\prime}$. To show that $y^{\prime} \in Y^{\prime}$ we need to show that $\operatorname{dim} F^{\prime}$ $<\operatorname{dim} G^{\prime}$.

Suppose $\operatorname{dim} F^{\prime}=\operatorname{dim} G^{\prime}$. Since $G^{\prime}$ is connected, $F^{\prime}=G^{\prime}$. Therefore $G=F \cdot \operatorname{ker} \varrho$. Since ker $\varrho$ commutes with $U, U C \mathrm{gFg}^{-1}$ for all $\mathrm{g} \in \mathrm{G}$. Also $\left(g F g^{-1}\right)(g y)=g F y$ is closed for all $g \in G$. Therefore $g y \in Y$ for all $g \in G$. Hence $\bar{\varrho}(Y) \subset Y^{\prime}$ if $Y \neq X$.

Proof of the main theorem 1.1. Let $\varrho, G^{\prime}, U^{\prime}=\left\{u_{\gamma}^{\prime}\right\}_{\mathrm{r} \in \mathbf{R}}, X^{\prime}, Y^{\prime}$ etc. be as defined earlier. Let $x^{\prime}=\bar{\varrho}(x)$. Then $x^{\prime} \in X^{\prime} \backslash Y^{\prime}$ by Lemma 6.16. By Corollary 5.6 and Theorem 6.1, there exists a compact subset $K^{\prime} \subset X^{\prime} \backslash Y^{\prime}$ such that for all $T>0$,

$$
(1 / T) \mathcal{\ell}\left\{t \in[0, T] \mid u_{r}^{\prime} x^{\prime} \in K^{\prime}\right\}>1-\varepsilon .
$$

Let $K=\bar{\varrho}^{-1}\left(K^{\prime}\right)$. Then $K$ is compact and by Lemma $6.16, K \subset X \backslash Y$. Now $u_{t} x \in K$ if and only if $u_{t}^{\prime} x^{\prime} \in K^{\prime}$. Hence for all $T>0$,

$$
(1 / T) \ell\left\{t \in[0, T] \mid u_{\imath} y \in K\right\}>1-\varepsilon .
$$

\section{Deductions}

(7.1) Corollary. Let $G$ be a connected reductive Lie group, $\Gamma$ be a lattice in $G$ and $U=\left\{u_{t}\right\}_{t \in \mathbb{R}}$ be a regular unipotent one-parameter subgroup of $G$. Let $x \in X=G / \Gamma$ and $F$ be the smallest Lie subgroup of $G$ containing $U$ such that $F x$ is closed. If $F$ is $a$ reductive group then the $U$-orbit through $x$ is uniformly distributed with respect to the $F$-invariant propability measure supported on $F x$.

Proof. By Theorem 2.3, $F_{x}$ is a lattice in $F$. By Lemma 4.2, $U$ is a regular unipotent one-parameter subgroup of $F$. Suppose if $F \neq G$ then $\operatorname{dim} F<\operatorname{dim} G$ and since 
$F / F_{x} \simeq F x$, the corollary will follow by induction. Now in the notation of the main theorem, we may assume that $x \in X \backslash Y$.

Let $\bar{X}=X \cup\{\infty\}$ be the one point compactification of $X$. For $T>0$ let $v_{T}$ be the measure on $\bar{X}$ such that for all continuous functions $f$ on $\bar{X}$,

$$
\int_{\mathrm{X}} f d v_{T}=\frac{1}{T} \int_{0}^{T} f\left(u_{t} x\right) d t .
$$

Note that the space of Borel probability measures on a compact second countable space is compact with respect to the weak* topology. Now to prove uniform distribution of the $\left\{u_{t}\right\}$-orbit through $x$, it is enough to prove that whenever for a sequence $T_{i} \rightarrow \infty$ the sequence of measures $v_{T_{i}}$ converges (in the weak* topology), the limit measure $v$ is supported on $X$ and is $G$-invariant.

First we claim that $v(Y \cup\{\infty\})=0$. Let $\varepsilon>0$ be given. By the main theorem, there exists a compact set $K \subset X \backslash Y$ such that for all $T>0$,

$$
(1 / T) \ell\left\{t \in[0, T] \mid u_{t} y \in K\right\}>1-\varepsilon .
$$

Now $\Omega=\bar{X} \backslash K$ is a neighbourhood of $Y \cup\{\infty\}$ and $v_{T}(\Omega)<\varepsilon$ for all $T>0$. Hence $v(\Omega)<\varepsilon$. This proves the claim.

It is easy to see that $v$ is $U$-invariant. Therefore there exist a partition of $X$ into $U$-invariant subsets $X_{c}, C \in \xi$, probability measures $\pi_{C}$ on $X_{C}$ and a probability measure $\pi$ on $\xi$ such that (a) for almost all $C \in \xi, \pi_{C}$ is $U$-ergodic invariant, and (b) for any measurable $A \subset X, A \cap X_{C}$ is measurable for almost all $C \in \xi$ and $v(A)$ $=\int_{\xi} \pi_{C}(A \cap C) d \pi(C)$ (cf. [2, Sect. 1.4]). Since $v(Y)=0$, we have $\pi_{C}(Y)=0$ for almost all $C \stackrel{\xi}{\xi}$. By Ratner's Theorem [19], the preceeding observation implies that for almost all $C, \pi_{C}$ is $G$-invariant. Hence $v$ is invariant under the action of $G$. This completes the proof.

(7.2) Remark. Corollary 1.2 is a particular case of Corollary 7.1.

Proof of Corollary 1.3. Let the notation be as in Sect. 6.14. Let $x \in X$ and $x^{\prime}=\bar{\varrho}(x) \in X^{\prime}$. Let $F$ be the smallest Lie subgroup of $G$ such that $U \subset F$ and $F x$ is closed. If $F^{\prime}$ is the smallest Lie subgroup of $G^{\prime}$ such that $U^{\prime} \subset F^{\prime}$ and $F^{\prime} x^{\prime}$ is closed then $F_{1}=\varrho(F)$. Now $X^{\prime}$ is compact. Therefore by Remark $6.4, F^{\prime}$ is reductive. Hence by the definition of $\varrho, F$ is reductive. Now apply Corollary 7.1 to complete the proof.

Proof of Corollary 1.4. Let the notations be as in Sect. 6.14. For $x \in X$ let $x^{\prime}, F$ and $F^{\prime}$ be defined as in the proof of Corollary 1.3. If $F^{\prime}$ is reductive then $F$ is reductive and we can apply Corollary 7.1 to complete the proof.

Otherwise by Proposition 3.1, $F^{\prime}$ is a unipotent subgroup of $G^{\prime}$ and $F^{\prime} x^{\prime}$ is compact. Since $F^{\prime}=\varrho(F)$, it follows that $F=C \cdot W$, where $C$ is a connected compact normal semisimple subgroup of $F$ and $W$ is the nilpotent radical of $F$. Therefore $U \subset W$ and $C \subset Z_{\mathrm{G}}(W)$. Since $W$ is normal in $F, R=\overline{W F}_{x}$ is a subgroup of $F$ and $\overline{W x}$ $=R^{0} x$. By a theorem of Auslander (applied to $F / F_{x}$ ), $R^{x}$ is a solvable group (cf. [18, Sect. 8.24]). From the structure of $F$ it is clear that $R^{0}$ is actually a nilpotent group and by the definition of $F, R^{0}=F$. Now the uniform distribution of the $U$-orbit through $x$ follows from a result of Green (cf. [13], see also [17, Theorem 5]) about flows on compact nilmanifolds. This completes the proof.

(7.3) Remark. Let $G=S L(3, \mathbb{R}), \Gamma$ be a lattice in $G$ and $U$ be any unipotent one parameter subgroup of $G$. Then using the method of this paper, it is not very difficult to verify the validity Conjecture 3 in this case. 
(7.4) Remark. Let $G=S O(n, 1), \Gamma$ be a lattice in $G$ and $L$ be a closed subgroup generated by unipotent one-parameter subgroups contained in $L$. In this case using the methods of $[15,7,8]$ and the method of the proof of the main theorem the author is able to verify Conjecture 1 (for $L$ in place of $U$ ), without using the classification of invariant measures involved in the proof of the above corollaries.

Acknowledgements. I am very grateful to S.G. Dani for having initiated me into this area. I thank him for a number of useful discussions we have had and for his help in writing this paper. I thank Gopal Prasad for clarifying my doubts regarding algebraic groups and explaining many of the results in great detail. I appreciate very much the encouragement he gave me.

\section{References}

1. Borel, A.: Introduction aux groupes arithmetiques. Publ. Inst. Math. Univ. Strasbourg XV. Paris: Hermann 1969

2. Dani, S.G.: Invariant measures of horospherical flows on noncompact homogeneous spaces. Invent. Math. 47, 101-138 (1978)

3. Dani, S.G., Smillie, J.: Uniform distribution of horocycle orbits for Fuchsian Groups. Duke Math. 51, 185-194 (1984)

4. Dani, S.G.: Dynamics of flows on homogeneous spaces: A survey. Proceedings of Coloquio de Systemas Dinamicos (Guanajuato, 1983). Aportaciones Mat. Notas Invest. 1, 1-30 (1985)

5. Dani, S.G.: On orbits of unipotent flows on homogeneous spaces. Ergodic Theory Dyn. Syst. 4, 25-34 (1984)

6. Dani, S.G.: On orbits of unipotent flows on homogeneous spaces II. Ergodic Theory Dyn. Syst. 6, 167-182 (1986)

7. Dani, S.G., Margulis, G.A.: Values of quadratic forms at primitive integral points. Invent. Math. 98, 405-424 (1989)

8. Dani, S.G., Margulis, G.A.: Orbit closures of generic unipotent flows on homogeneous spaces of $S L(3, \mathbf{R})$. Math. Ann. 286, 101-128 (1990)

9. Dani, S.G., Margulis, G.A.: Values of quadratic forms at integral points: an elementary approach. Enseign. Math. 36, 143-174 (1990)

10. Denker, M., Grillenberger, Ch., Sigmund, K.: Ergodic theory on compact spaces. (Lect. Notes Math., vol. 527) Berlin Heidelberg New York: Springer 1976

11. Fürstenberg, H.: The unique ergodicity of the horocycle flow. In: Beck, A. (ed.) Recent Advances in Topological Dynamics. (Lect. Notes Math., vol. 318, pp. 95-115) Berlin Heidelberg New York: Springer 1973

12. Garland, H., Raghunathan, M.S.: Fundamental domains for lattices in R-rank 1 semisimple Lie groups. Ann. Math. 92, 279-326 (1970)

13. Green, L.: Nilflows, measure theory. In: Flows on homogeneous spaces. Ann. Math. Studies. Auslander, L., Green, L., Hahn, F. (eds.). Princeton: Princeton Univ. Press 1963

14. Margulis, G.A.: Lie groups and ergodic theory. In: Avramov, L.L. (ed.) Algebra - Some Current Trends. Proceedings Varna 1986. (Lect. Notes Math., vol. 1352, pp. 130-146) Berlin Heidelberg New York: Springer 1988

15. Margulis, G.A.: Discrete subgroups and ergodic theory. In: Number theory, trace formulas and discrete subgroups, pp. 377-398. New York London: Academic Press 1989

16. Moore, C.C.: The Mautner phenomenon for general unitary representations. Pac. J. Math. 86, 155-169 (1980)

17. Parry, W.: Ergodic properties of affine transformations and flows on nilmanifolds. Am. J, Math. 91, 757-771 (1969)

18. Raghunathan, M.S.: Discrete subgroups of Lie groups. Berlin Heidelberg New York: Springer 1972

19. Ratner, M.: Invariant measures for unipotent translations on homogeneous spaces. Proc. Nat. Acad. Sci. USA 87, 4309-4311 (1990)

20. Steinberg, R.: Conjugacy classes in algebraic groups (Lect. Notes Math., vol. 366). Berlin Heidelberg New York: Springer 1974

21. Zimmer, R.J.: Ergodic theory and semisimple groups. Boston: Birkhäuser 1984

Note added in proof. Subsequent to writing this paper the author received a preprint from $M$. Ratner, where Conjecture I has been settled. 